\title{
Optimal Recharging Policies for Electric Vehicles
}

\author{
Timothy M. Sweda, Irina S. Dolinskaya, and Diego Klabjan \\ Department of Industrial Engineering and Management Sciences \\ Northwestern University
}

2145 Sheridan Rd.

Evanston, Illinois 60208, USA

E-mail: tsweda@u.northwestern.edu,dolira@northwestern.edu,d-klabjan@northwestern.edu

\begin{abstract}
Recharging decisions for electric vehicles require many special considerations due to battery dynamics. Battery longevity is prolonged by recharging less frequently and at slower rates, and also by not charging the battery too close to its maximum capacity. In this paper, we address the problem of finding an optimal recharging policy for an electric vehicle along a given path. The path consists of a sequence of nodes, each representing a charging station, and the driver must decide where to stop and how much to recharge at each stop. We present efficient algorithms for finding an optimal policy in general instances with deterministic travel costs and homogeneous charging stations, and also for two specialized cases. In addition, we develop two heuristic procedures that we characterize analytically and explore empirically. We further analyze and test our solution methods on model variations that include stochastic travel costs and nonhomogeneous charging stations.
\end{abstract}

Keywords: electric vehicles; optimal recharging policies; lot sizing; convex ordering cost 


\section{Introduction}

For drivers seeking to reduce their dependence on fossil fuels, battery electric vehicles (EVs) have become a practical and affordable alternative in recent years to conventional gasoline-powered vehicles. EVs are powered by electricity only, requiring no gasoline, and connect to the electrical grid to recharge. While the ability to plug in and recharge offers the potential for significant savings in fuel costs as well as other benefits, such as fewer greenhouse gas emissions and reduced dependence on foreign oil, there are still a number of obstacles to mass EV adoption.

One reason why many buyers are reluctant to purchase an EV is range anxiety (Klabjan and Sweda (2011)). The maximum range of an EV is less than that of a comparable gasoline-powered vehicle, and charging stations are scarcer than gasoline stations. Furthermore, if an EV runs out of charge along its route, there is no convenient method of recharging it from the side of the road. Spare batteries are prohibitively costly and bulky, in addition to being difficult to swap with the vehicle's depleted battery, and roadside charging services are either extremely limited or unavailable. As a result, EV drivers must be cautious when planning their routes to ensure that their vehicles do not run out of charge.

Unfamiliarity with battery dynamics also deters many potential EV purchasers. Recharging an EV's battery requires much more time than refueling a conventional vehicle, and unlike gasoline refueling, where the time required to refuel is roughly linearly related to the amount refueled (i.e., the refueling rate is constant), battery recharging occurs at a varying rate that depends on the charge level (Dearborn (2006)). In addition, charging too close to its maximum capacity (known as overcharging) can adversely affect the battery's lifespan, which is a major concern to EV owners since batteries are one of the most expensive and critical components of EVs. It is therefore important to understand the impacts of recharging decisions in order to minimize the costs of owning and operating an EV.

To overcome the aforementioned issues, this paper addresses the problem of finding an optimal recharging policy for an EV along a given path. The path consists of a sequence of nodes, each representing a charging station, and the vehicle must decide where to stop and how much to recharge at each stop. Whenever the vehicle stops to recharge, it incurs a fixed stopping cost, a charging cost based on the total amount it recharges, and an additional cost when the battery becomes overcharged. The goal is to minimize the total cost of recharging along the path, including all stopping, charging, and overcharging costs. Thus, optimal recharging policies provide the most favorable tradeoff between the number of times that the vehicle stops to recharge and the amount it recharges whenever it stops.

The model presented in this work represents the first effort in the literature to optimize recharging 
behavior specifically for EVs. We begin by identifying several properties of optimal recharging policies along a fixed path with deterministic travel costs and homogeneous charging stations. Using these properties, we develop efficient algorithms for finding an optimal recharging policy in the general case and in two specialized cases: when the vehicle can stop to recharge anywhere along the path (not just at prespecified nodes), and when the nodes with charging stations along the path are equidistant. We also describe two heuristic methods based on the properties of optimal paths that we use to obtain reasonable policies quickly, and we derive bounds on the quality of their solutions. To demonstrate the performance of these heuristics in practice, we implement them for highway and urban routes and conduct a numerical study to compare their solutions with those of optimal recharging policies. In addition, we formulate models that include stochastic travel costs and nonhomogeneous charging stations, and we provide detailed analyses and numerical experiments to illustrate how the solution approaches are affected.

The main contributions of this paper are: $(i)$ an efficient algorithm for obtaining an optimal recharging policy for the vehicle recharging problem; (ii) closed-form optimal policies for instances in which either charging capability is available continuously along the path or charging stations are equidistantly spaced; (iii) two heuristic methods that are easy to implement and yield reasonable recharging policies with little computational effort, along with bounds on their solution quality; (iv) a numerical study that demonstrates the actual performance of the heuristics for trips along both highway and urban routes, and $(v)$ insightful discussion and results (both analytical and numerical) of several different extensions to the model.

The remainder of the paper is organized as follows. Section 2 provides an overview of the existing literature on topics related to EV recharging. Section 3 describes the model studied in this work along with some properties of optimal recharging policies, and Section 4 details optimal and heuristic algorithms that can be used to obtain recharging policies under different scenarios. In Section 5, several possible extensions to the model are discussed along with how to find an optimal policy under such cases, and in Section 6 a case study implementation of the algorithms is demonstrated for the base model and its extensions. Lastly, Section 7 summarizes the conclusions and future directions of this research. Additional proofs and figures are presented in Appendices A1 and A2, respectively.

\section{Literature review}

The refueling problem for gasoline-powered vehicles, where drivers must decide at which nodes to refuel as well as how much to refuel in order to minimize the total cost of fuel, has been well studied. Khuller et al. 
(2007) and Lin et al. (2007) show that the optimal refueling policy along a fixed path can be solved easily with dynamic programming when fuel prices at each node are static and deterministic. For such a problem, the optimal decision at each node is always one of the following: do not refuel, refuel completely, or refuel just enough to reach the next node where refueling occurs. An algorithm for simultaneously finding the optimal path and refueling policy in a network is detailed by Lin (2008a), and some combinatorial properties of the optimal policies are explored by Lin (2008b). Specifically, it is proven that the problem of finding all-pairs optimal refueling policies reduces to an all-pairs shortest path problem that can be solved in polynomial time. However, all of the aforementioned analyses only consider fuel costs and not stopping or other costs. We include these additional costs in our analysis because they can comprise a significant portion of the total cost of traveling along a path and therefore can influence optimal recharging policies.

Several models have expanded on the vehicle refueling problem by introducing costs for stopping to refuel and traveling to refueling stations. A generic model for vehicle refueling is presented in Suzuki (2008) that attempts to capture such aspects, penalizing longer routes and routes with more refueling stops. Like other papers that study the vehicle refueling problem, it assumes that fuel prices at each station are static and deterministic. Approaches for finding optimal refueling policies when fuel prices are stochastic are given by Klampfl et al. (2008) and Suzuki (2009). Klampfl et al. (2008) use a forecasting model for predicting future fuel prices to generate parameters for a deterministic mixed integer program, and Suzuki (2009) presents a dynamic programming framework that is designed to grant drivers greater autonomy to select the stations where they refuel. These models are difficult to solve analytically, and the authors develop heuristics for obtaining reasonable solutions. In addition, just like the other models of the vehicle refueling problem, these ones do not include any costs that are analogous to battery overcharging costs for EVs. Sweda and Klabjan (2012) address this issue by introducing generalized charging cost functions, but some restrictive assumptions are required in order to perform insightful analysis. In this work, we use a tractable yet realistic charging cost function that enables us to easily find optimal recharging policies and develop a deeper understanding of such policies.

Overcharging costs, incurred when an EV's battery is charged near its maximum capacity, are important to consider when creating EV recharging policies for a number of reasons. Recharging an EV battery while it is already at a high state of charge takes place at a slower rate than when it is more depleted, and storing high levels of charge for prolonged periods of time can shorten the lifespan of the battery. A couple of models describing this relation can be found in Millner (2010) and Serrao et al. (2011). Overcharging also causes battery degradation due to greater stresses from being charged near full capacity and excess heat 
generated during recharging. In the refueling problem for conventional vehicles, the only main disadvantage of traveling with a full tank of fuel is the limited ability to take advantage of lower fuel prices further along the route. Optimal solutions tend to favor filling large quantities and making fewer stops (assuming that stopping costs are considered), but the opposite is true for optimal EV recharging policies.

To solve the problem of finding a path for an EV within a network with recharging considerations, a recent thread of research has taken an entirely different approach, having vehicles recharge via regenerative braking rather than by recharging at stations along their paths. As an EV decelerates, it can recapture some of its lost kinetic energy as electrical energy, which can then be used to recharge the battery. It is therefore possible in some cases for an EV's state of charge to increase while traveling rather than decrease, such as when the vehicle is coasting and braking downhill. Artmeier et al. (2010) model the problem of finding the most energy-efficient path for an EV in a network as a shortest path problem with constraints on the charge level of the vehicle, such that the charge level can never be negative and cannot exceed the maximum charge level of the battery. Edge weights are permitted to be negative to represent energy recapturing from regenerative braking, but no negative cycles exist. A simple algorithm for solving the problem is provided, and more efficient algorithms are presented by Eisner et al. (2011) and Sachenbacher et al. (2011). Eisner et al. (2011) show that the battery capacity constraints can be modeled as cost functions on the edges, and a transformation of the edge cost functions permits the application of Dijkstra's algorithm. The approach described by Sachenbacher et al. (2011) avoids the use of preprocessing techniques so that edge costs can be calculated dynamically, and it achieves an order of magnitude reduction in the time complexity of the algorithm from Artmeier et al. (2010). In practice, however, the amount of energy recovered by regenerative braking is insignificant compared with the amount that must be recharged at charging stations, and these papers do not model recharging decisions at nodes. Consequently, they also do not capture overcharging costs considered in the presented work.

One model type that is well suited for capturing overcharging costs is the inventory model with a convex ordering cost function. If the inventory corresponds to a vehicle's charge level and the ordering cost corresponds to the cost of recharging, then the convexity of the ordering cost function can be interpreted as the result of overcharging costs. Unfortunately, few papers in the literature have studied inventory models with convex ordering costs. The earliest of such models appeared in Bellman et al. (1955) and Karlin (1958), albeit with limited discussion. Three different models with general convex ordering costs are analyzed by Bulinskaya (1967) and include assumptions such as random delivery of orders, perishable inventory, and multiple orders with different delivery times, although closed-form optimal policies are not given. A model 
with a piecewise linear convex ordering cost function is studied in Henig et al. (1997), and Bhaskaran et al. (2010) characterize optimal inventory policies for a model with convex ordering costs in which excess demand may either be accepted (and backlogged) or rejected. However, these models all have stationary random demands, whereas the demands in our model (i.e., the energy consumptions between each pair of nodes) are deterministic and non-stationary. The models also assume that inventory levels are uncapacitated, which is not useful for modeling vehicle recharging policies since batteries are limited in the amount of energy that they can store. Atamtürk and Küçükyavuz (2005) study an inventory-capacitated lot-sizing model, but their model does not consider convex inventory ordering cost functions.

\section{$3 \quad$ Vehicle recharging problem}

In this paper, we study the following recharging problem for EVs. Consider an EV with battery capacity $q_{\text {max }}$ that must travel along a fixed path $P=(1, \ldots, n+1)$ consisting of a sequence of $n+1$ nodes. Charging stations are available at each of the first $n$ nodes $\left(\right.$ let $S_{P}=(1, \ldots, n)$ denote the sequence of nodes in $P$ that have charging stations), and the driver must decide how much to recharge at each station. The vehicle's charge level can never exceed the maximum capacity of the battery, and it can never drop below zero. We also do not allow the vehicle to discharge energy back to the grid. We let $q_{i}$ denote the charge level of the vehicle when it arrives at node $i$ and $h_{i}>0$ denote the amount of charge required to travel from node $i$ to node $i+1$. Then the set of feasible charging amounts, which we denote $\mathcal{A}_{i}\left(q_{i}\right)$, is $\mathcal{A}_{i}\left(q_{i}\right)=\left[\left(h_{i}-q_{i}\right)^{+}, q_{\max }-q_{i}\right]$. We assume that $h_{i} \leq q_{\max }$ for all $i \in S_{P}$ so that a feasible recharging policy exists.

Each time that the vehicle stops to recharge, it incurs a fixed stopping cost $s$. The stopping cost may include the time required to access the charging station, any fees charged by the station owner for allowing the driver to use the station, the cost of reducing the lifespan of the vehicle's battery by one charging cycle, and an additional penalty if the driver is averse to stopping frequently to recharge. The vehicle also incurs a recharging cost at a rate of $\gamma$ per unit of energy recharged $(\gamma \geq 0)$ plus an additional overcharging cost if the vehicle's charge level rises above $\alpha q_{\max }$, where $0<\alpha<1$. This threshold represents the point at which the charging voltage reaches its maximum value and the charging current begins to decrease (Dearborn (2006)). Thus, the overcharging cost takes into account both the additional time per unit of energy recharged (due to the decreasing current) and wear on the battery, each of which increase at an increasing rate with respect to the amount overcharged. We denote the overcharging cost as $f(x)$, where $x \in\left[0,(1-\alpha) q_{\text {max }}\right]$ is the amount by which the vehicle's charge level exceeds $\alpha q_{\max }$ after recharging and $f(\cdot)$ is convex and increasing with 
$f(0)=0$. If the vehicle's charge level already exceeds $\alpha q_{\max }$ when it stops to recharge, we discount the overcharging cost by $f\left(q_{i}-\alpha q_{\max }\right)$. Therefore, if we let $c(r, q)$ denote the cost of recharging $r$ at a node when the vehicle arrives at the node with charge level $q$, then we have

$$
c(r, q)=s I_{\{r>0\}}+\gamma r+\left[f\left(\left(q+r-\alpha q_{\max }\right)^{+}\right)-f\left(\left(q-\alpha q_{\max }\right)^{+}\right)\right]
$$

where $I_{\{r>0\}}$ is the indicator function that equals 1 if $r>0$ and 0 otherwise (see Figure 1 for an example illustration of $c(r, 0))$. Note that $c(r, q)=0$ whenever $r=0$. We assume that all charging stations are identical, which is reasonable since most public charging stations in existence today have similar hardware configurations (most are called Level 2 and recharge at 220 volts, with the exception of a few "fast charging," or Level 3, stations that recharge at 440 volts). Furthermore, under current state laws, charging station owners are prohibited from selling electricity by the kilowatt-hour (only the utility companies may do so), thereby mitigating the impacts of any regional or temporal variability in electricity prices on the charging cost rate.

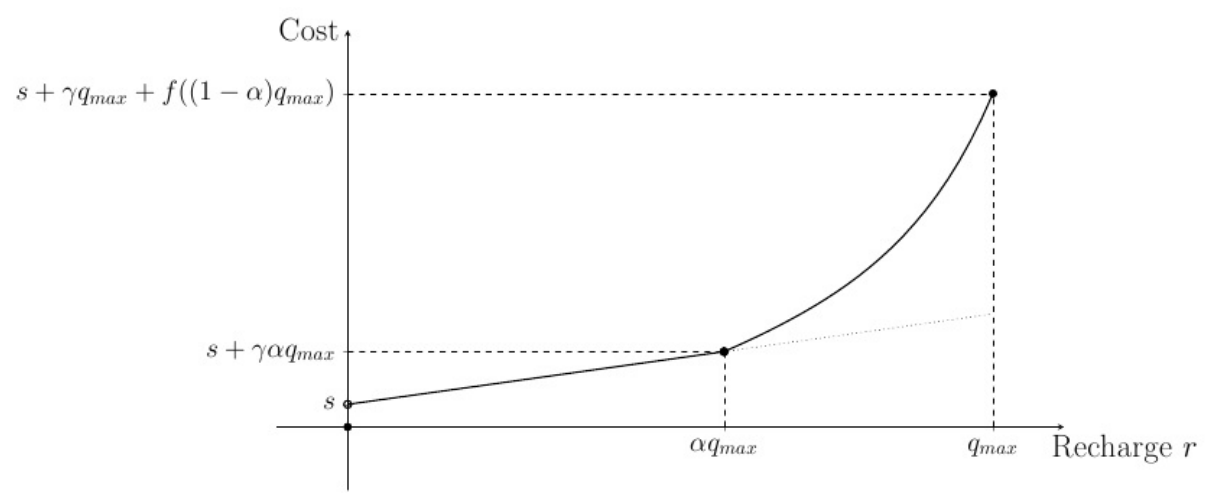

Figure 1: Sample illustration of $c(r, q)$ for $q=0$

Our objective is to minimize the total cost of recharging along the path $P$. We let $V_{i}\left(q_{i}\right)$ denote the value function, which represents the minimum cost of traveling to the end of $P$ from node $i$ starting with charge level $q_{i}$, and it can be defined recursively as

$$
V_{i}\left(q_{i}\right)=\min _{r_{i} \in \mathcal{A}_{i}\left(q_{i}\right)}\left\{c\left(r_{i}, q_{i}\right)+V_{i+1}\left(q_{i}+r_{i}-h_{i}\right)\right\}
$$

The first term within the brackets is the cost of recharging $r_{i}$ at node $i$ and the second term is the optimal recharging cost from the next node to the end of the path. We set $V_{n+1}(\cdot)=0$ and seek to evaluate $V_{1}(0)$, 
the minimum total cost when the vehicle's initial charge level at the beginning of the path is 0 .

\subsection{Properties of optimal recharging policies}

In this section, we establish properties of optimal recharging policies. We first define a recharging policy and its optimality. Let $\pi_{P}=\left[r_{1}, \ldots, r_{n}\right]$ be an ordered sequence, where $r_{i}$ represents the amount to recharge at node $i(i=1, \ldots, n)$. From (2), it follows that these recharging amounts must satisfy

$$
r_{i} \in \mathcal{A}_{i}\left(\sum_{j=1}^{i-1}\left(r_{j}-h_{j}\right)\right)
$$

for all $i \in S_{P}$ in order for $\pi_{P}$ to be a (feasible) recharging policy for $P$.

We denote by

$$
C\left(\pi_{P}\right)=\sum_{i \in S_{P}} c\left(r_{i}, \sum_{j=1}^{i-1}\left(r_{j}-h_{j}\right)\right)
$$

the total cost of policy $\pi_{P}=\left[r_{1}, \ldots, r_{n}\right]$. Our goal is to find an optimal policy for $P$ that minimizes the total cost. However, the action space $\mathcal{A}_{i}(\cdot)$ at each node is a continuous interval in $\mathbb{R}$. This can be problematic from an optimization perspective since there are infinitely many actions that must be considered. Thus, it would be beneficial to show that the action space can be reduced to a finite set without increasing the optimal cost. The following lemma establishes that there exists an optimal policy in which the vehicle only recharges when its charge level is zero (see Figure 2). (In the inventory theory, these policies are known as zero-inventory-ordering policies. It is interesting to point out that such policies are not optimal in the presence of a replenishment upper bound (Florian et al. (1980)), which is another indication that our problem is quite different.)

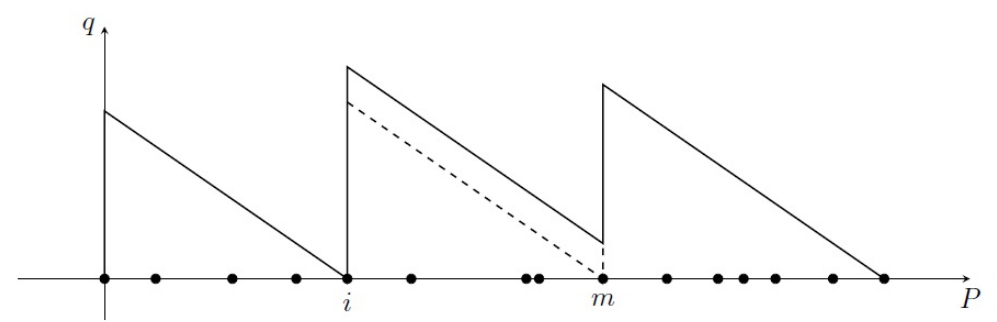

Figure 2: Original recharging policy (solid line) and alternate policy that only recharges when $q=0$ (dotted line), with charging station locations identified along the horizontal axis 
Lemma 1 Suppose $\pi_{P}^{*}=\left[r_{1}^{*}, \ldots, r_{n}^{*}\right]$ is an optimal recharging policy for $P$ in which

$$
m=\min \left\{\ell \in S_{P}: r_{\ell}^{*}>0, \sum_{j=1}^{\ell-1}\left(r_{j}^{*}-h_{j}\right)>0\right\}
$$

is the first node where the vehicle stops to recharge with a nonzero charge level and

$$
i=\max \left\{\ell \in\{1, \ldots, m-1\}: r_{\ell}^{*}>0\right\}
$$

is the previous node with a positive recharging amount in the policy. Then policy $\pi_{P}=\left[r_{1}, \ldots, r_{n}\right]$ defined as

$$
r_{\ell}= \begin{cases}\sum_{j=i}^{m-1} h_{j}, & \ell=i \\ r_{m}^{*}+\left(r_{i}^{*}-\sum_{j=i}^{m-1} h_{j}\right), & \ell=m \\ r_{\ell}^{*}, & \ell \in S_{P} \backslash\{i, m\}\end{cases}
$$

is also optimal.

Proof. See Appendix A1.

As a consequence of this lemma, the feasible action space at each node can be reduced to a discrete set without affecting the optimal cost. There exists an optimal policy such that if the vehicle recharges at some node $i \in S_{P}$, then the recharging amount equals $\sum_{j=i}^{m-1} h_{j}$ for some $m \in S_{P}$, and the vehicle will arrive at $m$ with zero charge level and recharge again. Thus, at any stop, the vehicle can recharge just enough to reach some later node, of which there are finitely many, and one such recharging policy is optimal. Without loss of generality, we introduce a property based on this result that will be used in our later analysis.

Property 1 Let $\pi_{P}=\left[r_{1}, \ldots, r_{n}\right]$ be a recharging policy for $P$. Then for all $i \in S_{P}, r_{i}>0$ if and only if $\sum_{j=1}^{i-1} r_{j}=\sum_{j=1}^{i-1} h_{j}$.

A vehicle obeying a recharging policy that satisfies Property 1 only recharges when its charge level is zero, and therefore never recharges more than necessary to reach its next stop. Although such a policy may seem impractical to implement, as drivers likely would be uncomfortable following a policy that leaves no room for error, an alternative interpretation of Property 1 is a model in which the vehicle's charge level is never allowed to drop below a given nonzero threshold value. This interpretation provides a more realistic setting without affecting the analysis (see Section 5.2 for further discussion of this topic).

The following two lemmas show that further reduction of the action space is possible, providing a lower 
bound on the amount that the vehicle recharges at each stop it makes.

Lemma 2 Let $P^{\prime}$ denote the path consisting of $P$ with an additional node at the beginning (node 0), and let $S_{P^{\prime}}=\left(0, S_{P}\right)$. If $\pi_{P}^{*}$ and $\pi_{P^{\prime}}^{*}$ are optimal recharging policies for $P$ and $P^{\prime}$, respectively, then $C\left(\pi_{P^{\prime}}^{*}\right) \geq$ $C\left(\pi_{P}^{*}\right)+\gamma h_{0}$

Proof. See Appendix A1.

One consequence of this lemma is that when an optimal recharging policy specifies an amount to recharge at a node that is less than $\alpha q_{\max }$, and increasing that amount by just enough to reach some further node along the path does not cause it to exceed $\alpha q_{\max }$ (see Figure 3), the total cost of the new policy is no greater than that of the original policy. This is formally stated in the following lemma.

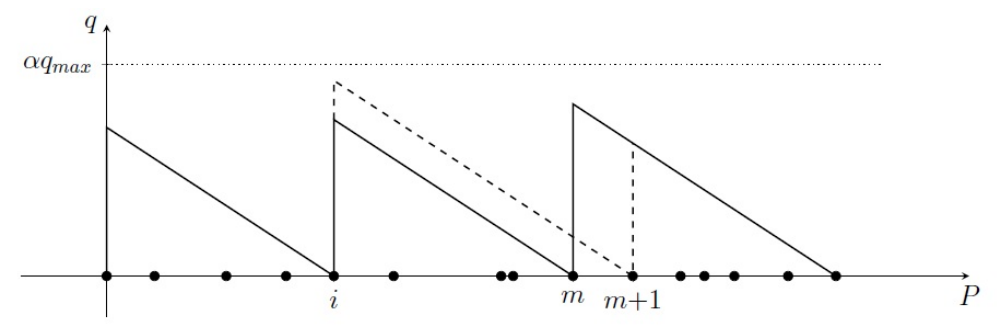

Figure 3: Original recharging policy (solid line) and alternate policy that recharges an additional amount at node $i$ without exceeding $\alpha q_{\max }$ (dotted line), with charging station locations identified along the horizontal axis

Lemma 3 Let $\pi_{P}^{*}=\left[r_{1}^{*}, \ldots, r_{n}^{*}\right]$ be an optimal recharging policy for $P$ where Property 1 holds. Suppose that $r_{i}^{*}>0$ for some node $i \in S_{P}$ and that

$$
m=\min \left\{\ell \in\{i+1, \ldots, n\}: r_{\ell}^{*}>0\right\}
$$

is the next node where the vehicle is recharged. If $r_{i}^{*}+h_{m} \leq \alpha q_{\max }$, then the policy $\pi_{P}=\left[r_{1}, \ldots, r_{n}\right]$ with

$$
r_{\ell}= \begin{cases}r_{i}^{*}+h_{m}, & \ell=i \\ 0, & \ell=m \\ r_{m}^{*}+r_{m+1}^{*}-h_{m}, & \ell=m+1 \\ r_{\ell}^{*}, & \ell \in S_{P} \backslash\{i, m, m+1\},\end{cases}
$$

where $r_{n+1}^{*}=r_{n+1}=0$, is also optimal.

Proof. See Appendix A1. 
We have now established several properties of optimal recharging policies that restrict the range of possible recharging amounts at each node to a discrete set. In the next section, we show how to apply these properties in order to find recharging policies under various sets of assumptions.

\section{Solution methods}

This section describes methods for obtaining recharging policies for EVs along a fixed path. We first find optimal recharging policies for general paths and two specific path types. We then analyze two heuristic methods and derive bounds on the costs of the resulting policies.

\subsection{Optimal policy algorithm for a general path}

The properties established in the previous section for optimal recharging policies can be used to design an algorithm capable of finding an optimal recharging policy for a given path $P$ efficiently. Although it is possible to solve the recursive expression given in equation (2) by imposing restrictions on the amount that can be recharged at each node, a disadvantage to this approach is that the value function must be calculated for every node and for multiple different charge levels. We instead propose a modified reaching procedure (see Algorithm 1) that computes the value function only when $q_{i}=0$ and only for nodes where the vehicle stops whenever the properties from Section 3.1 apply. A naive dynamic programming algorithm would use the fact that the vehicle recharges only when $q_{i}=0$ and scan all nodes along the path. Here, we present a more efficient version relying on Lemma 3.

In Algorithm 1, $U_{j}$ is the total cost of traveling from the beginning of the path to node $j$ and $N_{j}$ is the node from which the vehicle reaches node $j$ in an optimal recharging policy for the subpath $(1, \ldots, j)$ (i.e., the previous node where the vehicle stops to recharge in order to arrive at node $j$ with charge level $q_{j}=0$ )

for every $j \in S_{P}$. Beginning in line 2 , for all nodes $m$ satisfying $\alpha q_{\max }-h_{m}<\sum_{j=i}^{m-1} h_{j} \leq q_{\max }$ (i.e., the set of nodes requiring a charge level between $\alpha q_{\max }-h_{m}$ and $q_{\max }$ in order to be reached from $i$ ), if the sum of $U_{i}$ and the cost of recharging the exact amount required to reach node $m$ is less than $U_{m}$, then $U_{m}$ and $N_{m}$ are updated. The procedure is repeated for each possible value of $i$, and at the end, $U_{n+1}$ gives the total cost of an optimal policy while the $N_{j}$ values allow the recharging stops to be determined.

It is important to note that when the algorithm terminates, there may be some nodes $i$ for which $U_{i}$ (and also $N_{i}$ ) is not updated and therefore not equal to the optimal cost of traveling from the beginning of the path to node $i$. This is especially the case near the beginning of the path. For example, in the first iteration, 
if $m>1$ is the smallest index for which $U_{m}$ and $N_{m}$ are updated, then $U_{2}=\ldots=U_{m-1}=\infty$ at the end of the algorithm. The fact that $U_{i}=\infty$ at these nodes does not imply that stopping to recharge at any of them is infeasible, but rather that no optimal recharging policy (at least among the policies we consider) has recharging stops at these nodes.

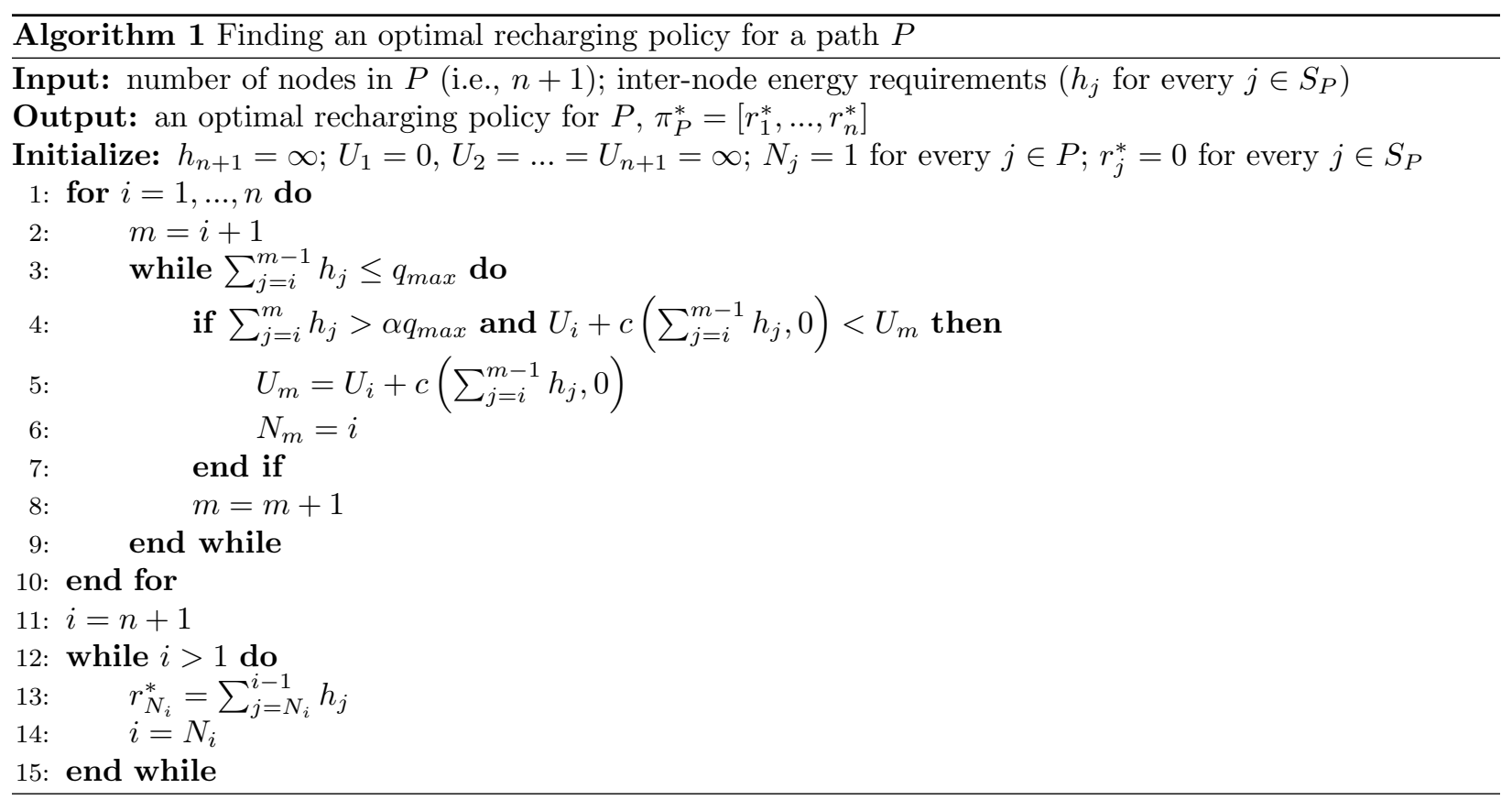

Finding an optimal recharging policy with Algorithm 1 is analogous to solving a shortest path problem on an auxiliary network in which there is an arc between every pair of nodes within $q_{\max }$ of each other and each arc has an associated cost equal to the cost of stopping to recharge at one node in order to reach the other. The advantage of Algorithm 1 over a more general shortest path algorithm is that it intelligently prunes arcs in the auxiliary network to reduce the overall network size. With fewer arcs to explore, it finds the shortest path (i.e., optimal recharging policy) more quickly and efficiently.

\subsection{Optimal policy for a path with continuous charging capability}

Next we consider the special case in which charging capability is available continuously along a path. In other words, a vehicle can stop to recharge anywhere along the path, not just at prespecified nodes. Although such a setting may be unrealistic, it can be used to compute a lower bound on the total cost of an optimal recharging policy for any given sequence of node locations. We redefine $r_{i}>0$ as the amount recharged at the $i^{\text {th }}$ stop for $i=1, \ldots, \xi$, where $\xi$ is the total number of stops. We also use $H$ to denote the total energy required to traverse the entire path $P$, and we redefine the encoding of path $P$ as the function 
$P(\cdot):[0,1] \rightarrow[0, H]$, with $P(x)=H x$, since $P$ is no longer a sequence of nodes. Because of this new definition, we no longer need to use $h_{i}$. We now explicitly define how continuous recharging policies differ from the recharging policies described previously.

Definition 1 Let $\rho_{P, \xi}=\left(\left[\lambda_{1}, \ldots, \lambda_{\xi}\right],\left[r_{1}, \ldots, r_{\xi}\right]\right)$ be a pair of ordered sequences satisfying $\lambda_{1}=0$ and

(i) $\lambda_{i}<\lambda_{i+1}$,

(ii) $\sum_{j=1}^{i} r_{j} \geq P\left(\lambda_{i+1}\right)$, and

(iii) $\left(P\left(\lambda_{i+1}\right)-\sum_{j=1}^{i-1} r_{j}\right)^{+} \leq r_{i} \leq q_{\max }-\left(\sum_{j=1}^{i-1} r_{j}-P\left(\lambda_{i}\right)\right)$

for all $i \in\{1, \ldots, \xi\}$ (where $\lambda_{\xi+1}=1$ ). Then $\rho_{P, \xi}$ is a (feasible) continuous recharging policy with $\xi$ stops for a path $P$, where $\lambda_{i}$ is the location of the $i^{\text {th }}$ recharging stop and $r_{i}$ represents the amount to recharge at the $i^{\text {th }}$ stop $(i=1, \ldots, \xi)$.

We denote by

$$
D\left(\rho_{P, \xi}\right)=\sum_{i=1}^{\xi} c\left(r_{i}, \sum_{j=1}^{i-1} r_{j}-P\left(\lambda_{i}\right)\right)
$$

the total cost of policy $\rho_{P, \xi}=\left(\left[\lambda_{1}, \ldots, \lambda_{\xi}\right],\left[r_{1}, \ldots, r_{\xi}\right]\right)$. Here, $i$ indexes the actual stops that the vehicle makes to recharge (as opposed to the nodes where the vehicle may stop in the previous definition of $c(\cdot)$ ).

By allowing the vehicle to recharge anywhere along the path instead of only at prespecified nodes, the total cost of an optimal continuous recharging policy is no greater than when the vehicle may only recharge at discrete intervals and provides a lower bound on the cost in the discrete case. The following theorems show how to easily find an optimal policy in the continuous case and compute such a bound for the discrete case. We first show that a continuous recharging policy of the form

$$
\rho_{P, \xi}^{*}=\left(\left[\lambda_{\ell}^{*}=(\ell-1) H / \xi: \ell=1, \ldots, \xi\right],\left[r_{\ell}^{*}=H / \xi: \ell=1, \ldots, \xi\right]\right)
$$

that recharges the same amount, $H / \xi$, at each stop is optimal for given $P$ and $\xi$.

Theorem 1 For given $P$ and $\xi$, the continuous recharging policy $\rho_{P, \xi}^{*}=\left(\left[\lambda_{\ell}^{*}=(\ell-1) H / \xi: \ell=1, \ldots, \xi\right],\left[r_{\ell}^{*}=\right.\right.$ $H / \xi: \ell=1, \ldots, \xi])$ is optimal.

Proof. See Appendix A1.

In addition to the structure of an optimal policy for a given number of stops, it is also desirable to know the number of stops that minimizes the total cost of an optimal recharging policy. To this end, we must evaluate 
the expression $\operatorname{argmin}_{\xi}\left\{D\left(\rho_{P, \xi}^{*}\right)\right\}$ to determine the optimal number of stops. This requires calculating $D\left(\rho_{P, \xi}^{*}\right)$ for every integer value of $\xi$ between $\left\lceil H / q_{\max }\right\rceil$ (the minimum feasible number of stops) and $\left\lceil H / \alpha q_{\max }\right\rceil$ (the minimum number of stops with no overcharging), inclusive. Note that for any $\xi>\left\lceil H / \alpha q_{\max }\right\rceil$, we have $D\left(\rho_{P, \xi}^{*}\right)=D\left(\rho_{P,\left\lceil H / \alpha q_{\max }\right\rceil}^{*}\right)+\left(\xi-\left\lceil H / \alpha q_{\max }\right\rceil\right) s$ since neither policy has any overcharging cost and there is no difference in the total amount recharged. In fact, for optimal policies of the form (3), the total amount recharged is the same for any $\xi$. To obtain a closed-form expression for the optimal number of stops, the following assumption must be made.

Assumption 1 In the expression for the cost of recharging given in (1), let $\gamma=0$ and $f(x)=k x$, where $k \geq 0$ is a constant.

Setting $\gamma=0$ is without loss of generality because every feasible recharging policy incurs a fixed cost of at least $\gamma H$, with optimal policies of the form (3) having a fixed cost of exactly $\gamma H$, and thus optimal recharging policies are not affected by adjusting $\gamma$. The use of a linear function for the overcharging cost $f(\cdot)$ simplifies some of our later calculations by allowing us to determine the optimal number of recharging stops as a function of the model parameters without having to create multiple different policies and evaluate their costs.

When Assumption 1 holds, the optimal number of stops depends on the ratio between the stopping cost, $s$, and the overcharging cost rate, $k$, of charging above the level $\alpha q_{\max }$. If the ratio is sufficiently small, then the minimum number of stops such that the amount recharged at each stop is less than $\alpha q_{\max }$ is optimal. As the ratio increases towards $\alpha q_{\max }$, it becomes optimal to recharge at least $\alpha q_{\max }$ at each stop, and if the ratio is equal to $\alpha q_{\max }$ or greater, then the amount recharged at each stop should be maximized in order to minimize the total number of stops. The following theorem states this in rigorous terms.

Theorem 2 Let Assumption 1 hold. For a path $P$, the value of $\xi$ that minimizes the total cost is

$$
\underset{\xi}{\operatorname{argmin}}\left\{D\left(\rho_{P, \xi}^{*}\right)\right\}= \begin{cases}\left\lceil\frac{H}{\alpha q_{\max }}\right\rceil, & \frac{s}{k}<H-\alpha q_{\max }\left\lfloor\frac{H}{\alpha q_{\max }}\right\rfloor \\ \left\lfloor\frac{H}{\alpha q_{\max }}\right\rfloor, & H-\alpha q_{\max }\left\lfloor\frac{H}{\alpha q_{\max }}\right\rfloor \leq \frac{s}{k}<\alpha q_{\max } \text { and }\left\lfloor\frac{H}{\alpha q_{\max }}\right\rfloor \geq\left\lceil\frac{H}{q_{\max }}\right\rceil \\ \left\lceil\frac{H}{q_{\max }}\right\rceil, & \text { otherwise. }\end{cases}
$$

Proof. See Appendix A1.

In this section, we have shown how to find an optimal continuous recharging policy for a given path. By calculating the ratio of the stopping cost parameter to the overcharging cost rate parameter, we can 
determine the optimal number of stops analytically, which we then use to determine the corresponding stopping locations and recharging amounts. The total cost of an optimal continuous recharging policy can therefore be computed quickly and in closed form. We use this solution in later sections to bound and compare the costs of other recharging policies.

\subsection{Optimal policy for a path with equidistant charging locations}

We now return to our original definition of a path, $P$, as a sequence of nodes, where $P=(1, \ldots, n+1)$. The vehicle may once again only recharge at nodes, but in this section we suppose that the nodes in $P$ are equidistant such that the distance between any pair of adjacent nodes is $h_{i}=h$ for all $i \in S_{P}$. We motivate this scenario as an intermediate case between general paths and paths with continuous charging capability. Although we enforce that recharging can only occur at nodes, the uniform spacing between nodes makes finding an optimal recharging policy easier than in the case of general paths. In fact, we show later in this section that a path with continuous charging capability is a limiting case of a path with equidistant charging locations, and thus the optimal policies in both cases are related.

Rather than use the same notation as before for optimal recharging policies for general paths, we define a new type of policy specifically for paths with equidistant charging locations, mimicking the continuous case.

Definition 2 Let $\sigma_{P, \xi}=\left(\left[\mu_{1}, \ldots, \mu_{\xi}\right],\left[r_{1}, \ldots, r_{\xi}\right]\right)$ be a pair of ordered sequences satisfying $\mu_{1}=1$ and

(i) $\mu_{i}<\mu_{i+1}$ (for all $\left.\mu_{i} \in S_{P}\right)$,

(ii) $\sum_{j=1}^{i} r_{j} \geq\left(\mu_{i+1}-1\right) h$, and

(iii) $\left(\left(\mu_{i+1}-1\right) h-\sum_{j=1}^{i-1} r_{j}\right)^{+} \leq r_{i} \leq q_{\max }-\left(\sum_{j=1}^{i-1} r_{j}-\left(\mu_{i}-1\right) h\right)$

for all $i \in\{1, \ldots, \xi\}$ (where $\mu_{\xi+1}=n+1$ ). Then $\sigma_{P, \xi}$ is a (feasible) equidistant recharging policy with $\xi$ stops for a path $P$, where $\mu_{i}$ is the node index of the $i^{\text {th }}$ recharging stop and $r_{i}$ represents the amount to recharge at the $i^{\text {th }}$ stop $(i=1, \ldots, \xi)$.

We let

$$
E\left(\sigma_{P, \xi}\right)=\sum_{i=1}^{\xi} c\left(r_{i}, \sum_{j=1}^{i-1} r_{j}-\left(\mu_{i}-1\right) h\right)
$$

denote the total cost of a policy $\sigma_{P, \xi}=\left(\left[\mu_{1}, \ldots, \mu_{\xi}\right],\left[r_{1}, \ldots, r_{\xi}\right]\right)$. An optimal policy over all policies with $\xi$ stops is defined in the same way as in the continuous case. 
When the number of recharging stops is fixed at $\xi$, then there exists a feasible recharging policy $\sigma_{P, \xi}=$ $\left(\left[\mu_{1}, \ldots, \mu_{\xi}\right],\left[r_{1}, \ldots, r_{n}\right]\right)$ with $r_{i}=\left\lceil\frac{n}{\xi}\right\rceil h$ at $n-\xi\left\lfloor\frac{n}{\xi}\right\rfloor$ of the stops and $r_{i}=\left\lfloor\frac{n}{\xi}\right\rfloor h$ at each of the remaining stops. The following theorem shows that this policy is also optimal.

Theorem 3 For given $P$ and $\xi$, where $h_{i}=h$ for all $i \in S_{P}$, the equidistant recharging policy $\sigma_{P, \xi}^{*}=$ $\left(\left[\mu_{1}^{*}, \ldots, \mu_{\xi}^{*}\right],\left[r_{1}^{*}, \ldots, r_{\xi}^{*}\right]\right)$ with $r_{1}^{*}=\ldots=r_{n-\xi\left\lfloor\frac{n}{\xi}\right\rfloor}^{*}=\left\lceil\frac{n}{\xi}\right\rceil h, r_{n-\xi\left\lfloor\frac{n}{\xi}\right\rfloor+1}^{*}=\ldots=r_{\xi}^{*}=\left\lfloor\frac{n}{\xi}\right\rfloor h$, and corresponding $\left[\mu_{1}^{*}, \ldots, \mu_{\xi}^{*}\right]$ that satisfy Property 1 is optimal.

Proof. See Appendix A1.

As with continuous optimal recharging policies, the optimal number of stops depends on the ratio between the stopping cost and overcharging cost rate when Assumption 1 holds. If the ratio is sufficiently small, then the minimum number of stops such that the amount recharged at each stop is less than $\alpha q_{\max }$ is optimal. The optimal number of stops decreases as the ratio increases, crossing different thresholds until it equals the minimum possible number of stops. The next theorem shows how to find the number of stops that minimizes the total cost of an optimal equidistant recharging policy in rigorous terms.

Theorem 4 Let Assumption 1 hold. For a path $P$ with $h_{i}=h$ for all $i \in S_{P}$, if $\sigma_{P, \xi}^{*}$ is an optimal equidistant recharging policy with $\xi$ stops and $y=\left\lfloor\alpha q_{\max } / h\right\rfloor$ (i.e., $y$ is the largest integer multiple of $h$ such that $\left.y h \leq \alpha q_{\max }\right)$, then the value of $\xi$ that minimizes the total cost of such a policy is

$$
\underset{\xi}{\operatorname{argmin}}\left\{E\left(\sigma_{P, \xi}^{*}\right)\right\}= \begin{cases}\left\lceil\frac{n}{y}\right\rceil, & \frac{s}{k}<n h-\left\lfloor\frac{n}{y}\right\rfloor \alpha q_{\max } \\ \left\lfloor\frac{n}{y}\right\rfloor, & n h-\left\lfloor\frac{n}{y}\right\rfloor \alpha q_{\max } \leq \frac{s}{k}<\alpha q_{\max } \text { and }\left\lfloor\frac{n}{y}\right\rfloor \geq\left\lceil\frac{n}{\left\lfloor q_{\max } / h\right\rfloor}\right\rfloor \\ \left\lceil\frac{n}{\left\lfloor q_{\max } / h\right\rfloor}\right\rceil, & \text { otherwise }\end{cases}
$$


if $\frac{n}{y+1}>\left\lfloor\frac{n}{y}\right\rfloor$ and

$$
\begin{aligned}
& \begin{cases}\left\lceil\frac{n}{y}\right\rceil, & \frac{s}{k}<\left(n-y\left\lfloor\frac{n}{y}\right\rfloor\right)\left((y+1) h-\alpha q_{\max }\right) \\
\left.\mid \frac{n}{-}\right\rfloor, & \left(n-y\left\lfloor\frac{n}{y}\right\rfloor\right)\left((y+1) h-\alpha q_{\max }\right) \leq \frac{s}{k}<y\left((y+1) h-\alpha q_{\max }\right)\end{cases} \\
& \text { and }\left\lfloor\frac{n}{y}\right\rfloor \geq\left\lceil\frac{n}{\left\lfloor q_{\max } / h\right\rfloor}\right\rceil \\
& y\left((y+1) h-\alpha q_{\max }\right) \leq \frac{s}{k}<\alpha q_{\max }-\left((y+1)\left\lceil\frac{n}{y+1}\right\rceil-n\right) \text {. } \\
& \left(\alpha q_{\max }-y h\right) \text { and }\left\lceil\frac{n}{y+1}\right\rceil \geq\left\lceil\frac{n}{\left\lfloor q_{\max } / h\right\rfloor}\right\rceil \\
& \alpha q_{\max }-\left((y+1)\left\lceil\frac{n}{y+1}\right\rceil-n\right)\left(\alpha q_{\max }-y h\right) \leq \frac{s}{k}<\alpha q_{\max } \\
& \text { and }\left\lfloor\frac{n}{y+1}\right\rfloor \geq\left\lceil\frac{n}{\left\lfloor q_{\max } / h\right\rfloor}\right\rceil \\
& \left\lceil\frac{n}{\left\lfloor q_{\max } / h\right\rfloor}\right\rceil, \quad \text { otherwise }
\end{aligned}
$$

if $\frac{n}{y+1} \leq\left\lfloor\frac{n}{y}\right\rfloor$.

Proof. See Appendix A1.

In a similar manner as for optimal continuous recharging policies, we have shown how to find an optimal equidistant recharging policy as well as the number of stops that minimizes the total cost of such a policy. It is useful to note that the case of continuous charging capability along the path is a limiting instance of the case with equidistant charging locations in which the distance between nodes goes to 0 . Because of the consistent path structure in each case, manageable closed-form threshold recharging policies exist, but this is not necessarily true for general paths. In the next section, we discuss heuristic methods that build on the special cases discussed in this section and impose consistency in their recharging policies despite possible irregularities in the distances between nodes for an arbitrary path.

\subsection{Heuristic solution methods}

So far, we have examined methods for finding optimal recharging policies. We have shown how to obtain optimal continuous and equidistant recharging policies by first solving a closed-form expression to determine the optimal number of stops, and then using that number of stops to construct an optimal policy. For general paths, we have presented an $O\left(n^{2}\right)$ algorithm that uses forward recursion to identify the nodes where the vehicle should stop to recharge. Although this last approach is extremely efficient and minimizes the total 
cost, the resulting policy can be unstructured and difficult to understand at a glance. An EV driver may prefer a simpler rule-of-thumb strategy that can be determined quickly with less computation. For that reason, we have developed two heuristic methods for creating reasonable recharging policies with intuitive interpretation and limited computational effort. These represent possible recharging behaviors that an EV driver is likely to follow without the assistance of an in-vehicle telematics system.

In Section 3.1, we established a range of possible actions at each recharging stop. Subsequently, from our analysis of optimal continuous and equidistant recharging policies in Sections 4.2 and 4.3, respectively, we found that there exist thresholds for when the vehicle should never overcharge and when it should overcharge to minimize its total number of stops. These observations motivate the two heuristics presented below.

\subsubsection{Heuristic 1: Avoid overcharging}

Our first heuristic, outlined in Algorithm 2, yields a policy in $O(n)$ time in which the vehicle minimizes its total number of stops while avoiding overcharging whenever possible. In the algorithm, $\hat{r}_{j}$ denotes the recharging amount at node $j$. The procedure finds the maximum value $m$ such that $\sum_{j=i}^{m-1} h_{j} \leq \alpha q_{m a x}$ (i.e., the charge level required at node $i$ to reach node $m$ is no greater than $\alpha q_{\text {max }}$ ), or if no such value exists, then $m=i+1$.

Note that the vehicle overcharges only when consecutive charging stations are sufficiently far apart (i.e., when $h_{i}>\alpha q_{\max }$ for some $\left.i \in S_{P}\right)$. Otherwise, it will never overcharge but still try to minimize the number of times that it stops to recharge. This heuristic method is most preferable when a driver is primarily concerned with preserving battery health and would rather stop more frequently to recharge than incur overcharging costs.

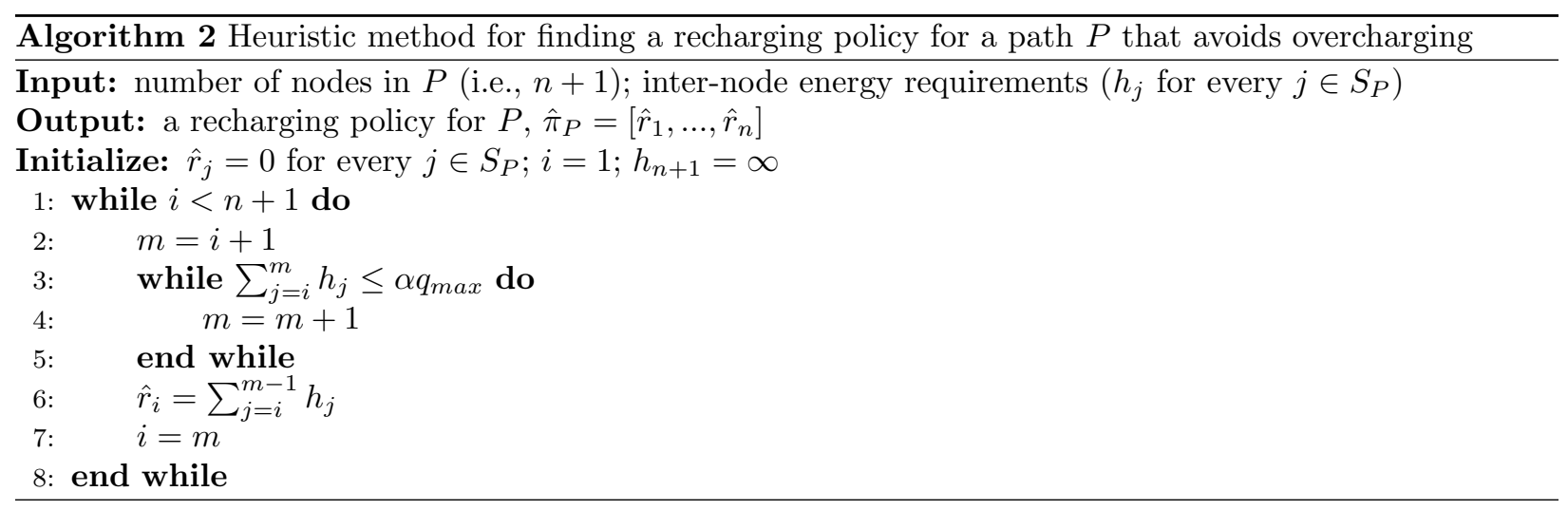

In the following two lemmas, we derive bounds on the number of stops and total cost of the policy obtained using Algorithm 2. We let $H=\sum_{i \in S_{P}} h_{i}$ denote the total energy required to travel along the path 
Lemma 4 Let $\hat{\pi}_{P}=\left[\hat{r}_{1}, \ldots, \hat{r}_{n}\right]$ be the recharging policy obtained using Algorithm 2 for the path $P$. Also let $\hat{\xi}=\sum_{j \in S_{P}} I_{\left\{\hat{r}_{j}>0\right\}}$ be the number of recharging stops in $\hat{\pi}_{P}$. Then

$$
\hat{\xi} \leq\left\lceil\frac{2 H}{\alpha q_{\max }}\right\rceil
$$

and this bound is tight.

Proof. See Appendix A1.

We use this result in the next theorem to calculate the maximum ratio between the cost of the policy from Algorithm 2 and the cost of an optimal recharging policy. The performance of this algorithm in practice is demonstrated later numerically in Section 6.1.

Theorem 5 Let $\pi_{P}^{*}=\left[r_{1}^{*}, \ldots, r_{n}^{*}\right]$ be an optimal recharging policy for the path $P$, and let $\hat{\pi}_{P}=\left[\hat{r}_{1}, \ldots, \hat{r}_{n}\right]$ be the recharging policy obtained using Algorithm 2. Then the cost of $\hat{\pi}_{P}$ relative to that of $\pi_{P}^{*}$ is bounded by

$$
\frac{C\left(\hat{\pi}_{P}\right)}{C\left(\pi_{P}^{*}\right)} \leq \frac{2}{\alpha}+\frac{q_{\max }}{H}
$$

Proof. See Appendix A1.

In the result of Theorem 5, the term $q_{\max } / H$ represents a correction factor for the ceiling function in the numerator of expression (18). As $H \rightarrow \infty$, the effect of the ceiling function becomes negligible and the ratio simply becomes $2 / \alpha$, which is also equal to the maximum ratio between the number of stops in the two policies.

\subsubsection{Heuristic 2: Minimize number of stops}

Our second heuristic seeks to minimize the total number of stops. As shown in Sections 4.2 and 4.3 for optimal continuous and equidistant recharging policies, as the stopping cost increases relative to the cost rate of overcharging, it becomes desirable to minimize the number of times that the vehicle stops to recharge. A method for obtaining such a policy is detailed in Algorithm 3. Like Algorithm 2, this algorithm also runs in $O(n)$ time. It finds the maximum value $m$ such that $\sum_{j=i}^{m-1} h_{j} \leq q_{\max }$ (i.e., the charge level required at node $i$ to reach node $m$ is no greater than $\left.q_{\max }\right)$.

In a recharging policy produced by this procedure, the vehicle minimizes its total number of recharging stops and, whenever it stops, always recharges enough to travel as far as it can without having to stop again. 
This heuristic performs best when overcharging costs are relatively low or the stopping cost is relatively high.

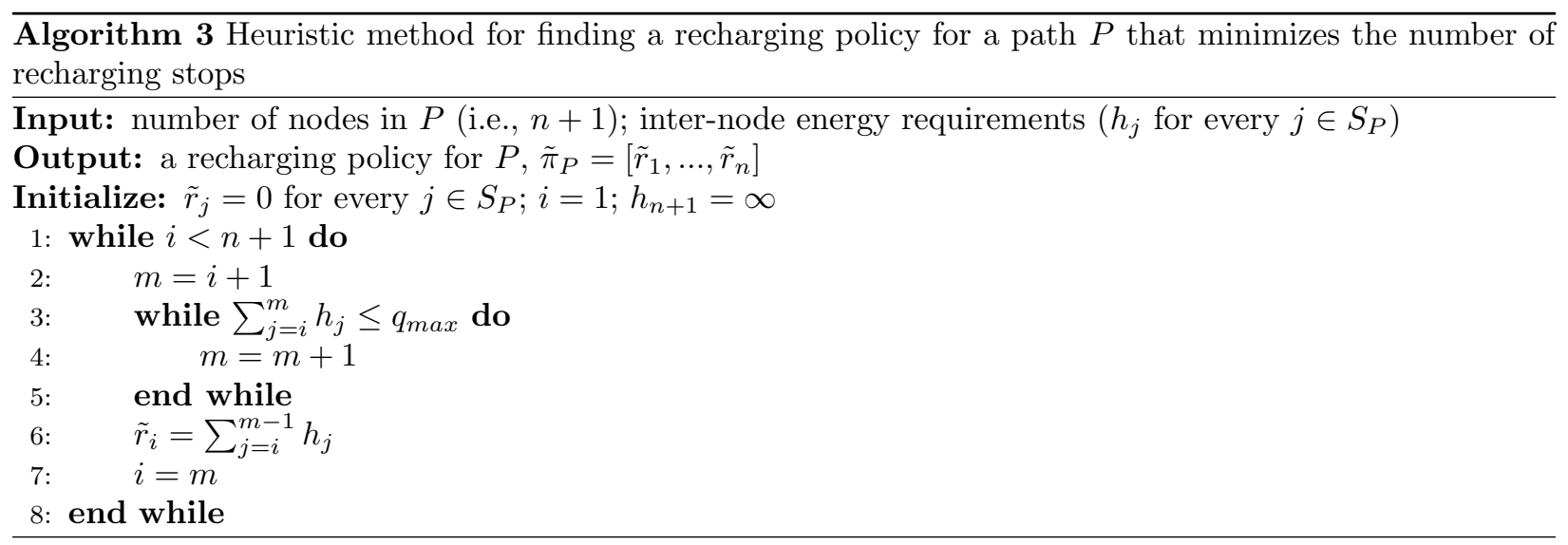

We next derive an upper bound on the total cost of a policy obtained using Algorithm 3 relative to the cost of an optimal recharging policy. (Numerical experiments are presented in Section 6.1 to demonstrate the practical performance of the algorithm.) In order to obtain a closed-form expression for the bound, we use Assumption 1 in our analysis.

Theorem 6 Let Assumption 1 hold. Let $\pi_{P}^{*}=\left[r_{1}^{*}, \ldots, r_{n}^{*}\right]$ be an optimal recharging policy for the path $P$, and let $\tilde{\pi}_{P}=\left[\tilde{r}_{1}, \ldots, \tilde{r}_{n}\right]$ be the recharging policy obtained using Algorithm 3. Then the cost of $\tilde{\pi}_{P}$ relative to that of $\pi_{P}^{*}$ is bounded by

$$
\frac{C\left(\tilde{\pi}_{P}\right)}{C\left(\pi_{P}^{*}\right)} \leq \begin{cases}1+(1-\alpha)\left(\frac{H / \alpha q_{\max }}{\left[H / \alpha q_{\max }\right.}\right)\left(\frac{\alpha q_{\max }}{s / k}\right), & s / k<\alpha q_{\max } \\ 1 / \alpha, & s / k \geq \alpha q_{\max } \text { and } H-\left\lceil\frac{H}{q_{\max }}\right\rceil \alpha q_{\max } \leq 0 \\ 1+\frac{\alpha q_{\max }}{H}, & s / k \geq \alpha q_{\max } \text { and } H-\left\lceil\frac{H}{q_{\max }}\right\rceil \alpha q_{\max }>0 .\end{cases}
$$

Proof. See Appendix A1.

When the stopping cost is sufficiently high and $s / k \geq \alpha q_{\text {max }}$, the goals of the optimal and heuristic policies are both to minimize the total number of stops. Because their goals are aligned, the two policies perform similarly, and the total cost of the heuristic policy is close to that of an optimal recharging policy. The bounding ratio $1 / \alpha$ applies for larger values of $\alpha$ whereas the ratio $1+\alpha q_{\max } / H$ applies for smaller values of $\alpha$, and both bounds are nearly equal to 1 for their respective $\alpha$ values. If the stopping cost is low, then the heuristic method performs quite poorly, and the value of the bounding ratio approaches $\infty$ as 
$s / k \rightarrow 0$.

\section{Model extensions}

We now consider several extensions to the model presented in Section 3. In particular, we examine how the analysis is affected when $(i)$ the vehicle's initial charge level is nonzero (Section 5.1); (ii) the arc travel costs $h_{i}$ are stochastic (Section 5.2); and (iii) the charging stations at each node are not identical (Section 5.3). We also conduct numerical experiments in Section 6 to illustrate how the heuristics perform under these revised scenarios.

\subsection{Initial charge level}

Suppose that instead of the vehicle having zero initial charge, it has some positive initial charge level $q_{1}>0$ and the objective is to evaluate $V_{1}\left(q_{1}\right)$ using equation (2). Observe that if $q_{1} \geq h_{1}$, then the vehicle is not required to recharge right away and can make its first recharging stop at some node further along the path. Because $q_{1}$ is an exogenous parameter, the vehicle's charge level when it first stops to recharge may be nonzero. By Lemma 1, however, there exists an optimal policy in which the vehicle's charge level is zero at all subsequent stops. We introduce a new property based on this observation.

Property 2 Let $\pi_{P}=\left[r_{1}, \ldots, r_{n}\right]$ be a recharging policy for $P$, and let

$$
i=\min \left\{\ell \in S_{P}: r_{\ell}>0\right\}
$$

be the first node where the vehicle stops to recharge. Then for all $\ell \in(i+1, \ldots, n), r_{\ell}>0$ if and only if $q_{1}+\sum_{j=1}^{\ell-1} r_{j}=\sum_{j=1}^{\ell-1} h_{j}$.

Property 2 is similar to Property 1 except that the vehicle's first recharging stop may occur when its battery is not yet empty. To determine where the first stop takes place, we consider the following lemma. It establishes that when the vehicle's initial charge level is nonzero, there exists an optimal policy satisfying Property 2 in which the vehicle delays its first recharging stop as much as possible.

Lemma 5 Let

$$
m=\max \left\{\ell \in S_{P}: q_{1}-\sum_{j=1}^{\ell-1} h_{j} \geq 0\right\}
$$


denote the furthest node along the path that the vehicle can reach without recharging. Suppose $\pi_{P, q_{1}}^{*}=$ $\left[r_{1}^{*}, \ldots, r_{n}^{*}\right]$ is an optimal recharging policy for $P$ when the vehicle's initial charge level is $q_{1}$, where $\pi_{P, q_{1}}^{*}$ satisfies Property 2, and let

$$
i=\min \left\{\ell \in\{1, \ldots, m-1\}: r_{\ell}^{*}>0\right\}
$$

denote first node where the vehicle stops to recharge. Then policy $\pi_{P, q_{1}}=\left[r_{1}, \ldots, r_{n}\right]$ defined as

$$
r_{\ell}= \begin{cases}0, & \ell=i \\ r_{i}^{*}, & \ell=m \\ r_{\ell}^{*}, & \ell \in S_{P} \backslash\{i, m\}\end{cases}
$$

is also optimal.

Proof. See Appendix A1.

It follows from Lemma 5 that if $m$ is the furthest node from the beginning of the path that the vehicle can reach without recharging, then

$$
V_{1}\left(q_{1}\right)=V_{m}\left(q_{1}-\sum_{j=1}^{m-1} h_{j}\right)=V_{m}(0)-\gamma\left(q_{1}-\sum_{j=1}^{m-1} h_{j}\right)-f\left(\left(q_{1}-\sum_{j=1}^{m-1} h_{j}\right)^{+}\right)
$$

In other words, the value of having a charge level of $q_{1}$ at the beginning of the path is equal to the value of starting from node $m$ with an initial charge level of zero minus the value of any remaining charge amount when the vehicle does not stop to recharge before $m$. The stopping cost $s$ is not included since it is incurred at $m$ whether the vehicle's charge level is 0 or $q_{1}-\sum_{j=1}^{m-1} h_{j}$ (as either is less than $h_{m}$ ). The quantity $V_{m}(0)$ can be computed using Algorithm 1, and thus having a nonzero initial charge level can be handled with direct extension of the preceding analysis and does not require new solution techniques.

\subsection{Stochastic travel costs}

In an actual setting, a driver may not know the exact $h_{i}$ values due to variations caused by weather, traffic, and other factors. Let $H_{i}$ denote the random variable representing the amount of charge required to travel from node $i$ to node $i+1$, where $E\left[H_{i}\right]=h_{i}$ for all $i \in S_{P}$. Note that under such a setting, it is possible for the vehicle to arrive at a node with a negative charge level (i.e., $q_{i}<0$ ). The physical interpretation of such an occurrence is that the vehicle ran out of charge before reaching the node and needed to be towed (or pushed, in the most unfortunate case) the rest of the way. Let $\beta>0$ denote the penalty cost per unit of 
charge below zero that the vehicle has upon arrival at a node (equivalent to stockout costs in the inventory literature). The term $\beta\left(-q_{i}\right)^{+}$therefore represents the cost of moving the vehicle from the spot after node $i$ where it runs out of charge to the charging station at node $i+1$, or it equals 0 if the vehicle does not run out of charge. Then the value function representation becomes

$$
V_{i}\left(q_{i}\right)=\min _{r_{i} \in \mathcal{A}_{i}\left(q_{i}\right)}\left\{\beta\left(-q_{i}\right)^{+}+c\left(r_{i}, q_{i}^{+}\right)+E\left[V_{i+1}\left(q_{i}^{+}+r_{i}-H_{i}\right)\right]\right\}
$$

where $V_{n+1}\left(q_{n+1}\right)=\beta\left(-q_{n+1}\right)^{+}$. (Alternatively, the term $\beta\left(-q_{i}\right)^{+}$could be replaced with or added to a scalar representing a fixed penalty, such as a towing fee, for arriving at a node with a negative charge level. However, such a formulation is more difficult to relate to the inventory literature.) Because of the penalty associated with arriving at a node with a negative charge level, it may not be optimal to recharge $r_{i}=\sum_{j=i}^{m-1} E\left[H_{j}\right]-q_{i}$ for some $m>i$ (such that the vehicle's expected charge level upon arrival at node $m$ is zero). Instead, it may be preferential for the recharging amount to be greater so that the likelihood of the vehicle arriving at node $m$ with a negative charge level is reduced.

Therefore, an optimal policy with stochastic travel costs attempts to maintain a buffer in the vehicle's charge level to account for the penalty of arriving at a node with a negative charge level. This buffer could be fixed to a certain percentage of $q_{\max }$ or a variable level that depends on the distance between planned recharging stops and uncertainty in travel costs. Furthermore, the vehicle's actual recharging stops will depend on the realized $H_{i}$ values. Property 1 does not apply in this section since the $H_{i}$ variables are not deterministic, and thus, the recharging amount at each node does not uniquely determine the next node at which the vehicle stops to recharge.

We conduct a detailed numerical study in Section 6.3 where we implement a model with stochastic travel costs to examine the comparative performance of our three algorithms. We also conduct a sensitivity analysis on the parameter inputs to the model and test several fixed buffer strategies.

\subsection{Nonhomogeneous charging stations}

In the preceding analysis, the charging stations at each node are assumed to be identical. However, some stations may be configured to allow drivers to recharge their vehicles at faster rates, while others may be at locations that provide diversions so that drivers do not mind waiting as much. We can model this by creating a separate charging cost function $c_{i}(r, q)$ for each node with associated parameters $s_{i}, \gamma_{i}$, and $f_{i}(\cdot)$, 
where

$$
\left.c_{i}(r, q)=s_{i} I_{r>0}+\gamma_{i} r+\left[f_{i}\left(\left(q+r-\alpha q_{\max }\right)^{+}\right)-f_{i}\left(q-\alpha q_{\max }\right)^{+}\right)\right] .
$$

The value function representation is thus

$$
V_{i}\left(q_{i}\right)=\min _{r_{i} \in \mathcal{A}_{i}\left(q_{i}\right)}\left\{c_{i}\left(r_{i}, q_{i}\right)+V_{i+1}\left(q_{i}+r_{i}-h_{i}\right)\right\}
$$

Because the charging stations are no longer identical, it may be desirable to recharge extra amounts at charging stations with favorable charging cost functions in order to minimize recharging activities at more costly stations. In fact, as proven in Sweda and Klabjan (2012), it is optimal for the vehicle to recharge fully at a charging station with a sufficiently low charging cost function, and at charging stations with high charging costs the vehicle should recharge the bare minimum required to reach the next stop. It follows that if the vehicle stops at node $i$ to recharge and its next recharging stop is at node $j$, then the optimal amount to recharge at node $i$ is one of the following:

(i) $\sum_{\ell=i}^{j-1} h_{\ell}-q_{i}$, the minimum amount required to reach node $j$;

(ii) $q_{\max }-q_{i}$, the maximum feasible amount; or

(iii) some amount between $(i)$ and $(i i)$.

In the most general case, solving for the amount (iii) could require minimization of a nonconvex function. However, as long as the overcharging cost functions at each node are of a similar functional form (which is often the case in practice), the minimization problem is much simpler.

It is evident that the vehicle may recharge even when its charge level is nonzero in the case of nonhomogeneous charging stations. Nevertheless, the optimal action space is discrete and the state space also reduces to a discrete set, and therefore an optimal policy can be found in $O\left(n^{2}\right)$ time using a backward recursive algorithm.

\section{Numerical results}

To compare the actual performance of the two heuristic methods against the exact algorithm for finding an optimal recharging policy, we implemented Algorithms 1, 2, and 3 for a highway route, using data for U.S. Interstate 90 (I-90), and also for simulated urban routes. We are primarily interested in the solution quality of the heuristics as opposed to their runtime since Algorithm 1, which yields an optimal policy, is extremely 
efficient (requiring less than one second). However, the optimal policy may not be readily apparent to an EV driver, whereas policies obtained from the heuristic algorithms are more intuitive and easier to understand.

We consider the portion of I-90 in the eastbound direction that begins in the city of Chicago, Illinois, and ends in Boston, Massachusetts, spanning a total of approximately 1,000 miles. In particular, we study a set of 22 trips ranging in length from 100 to 300 miles and having start and end points among 13 major cities (see Table 1). Each trip is one that an EV driver could reasonably complete in a day. (We also examined trips longer than 300 miles; however, as we explain in Section 6.1, their results did not provide additional insights.) To emulate both highway and urban driving, multiple sets of node locations were generated. The 208 node locations for the highway configuration were determined by identifying existing exits along the highway leading to rest areas, towns, or other places where charging stations might feasibly be located. For urban driving, possible node locations were located every 0.5 miles along the entire path (representing intersections), and among these, 10\% (200) were randomly selected to be the actual recharging node locations. Thus, charging station locations in an urban setting have less of a clustering effect one usually sees on highways. See Figure 4 for the node locations along the highway and urban routes.

\begin{tabular}{|r|c|c|c|c|c|c|c|c|c|c|c|c|c|}
\hline Origin City & Abbr. & Mile Marker & \multicolumn{7}{|c|}{ Destination (Distance in mi.) } \\
\hline & & & SB & FW & Tol & Cle & Erie & Buf & Roc & Syr & Alb & Spr & Bos \\
\hline Chicago, IL & Chi & 0 & 100 & 171 & 244 & & & & & & & & \\
\hline Indianapolis, IN & Ind & 45 & & 127 & 199 & & & & & & & & \\
\hline South Bend, IN & SB & 100 & & & 143 & 257 & & & & & & & \\
\hline Fort Wayne, IN & FW & 171 & & & & 186 & 275 & & & & & & \\
\hline Toledo, OH & Tol & 244 & & & & 114 & 203 & 295 & & & & & \\
\hline Cleveland, OH & Cle & 357 & & & & & & 182 & 245 & & & & \\
\hline Erie, PA & Erie & 447 & & & & & & & 156 & 229 & & & \\
\hline Buffalo, NY & Buf & 539 & & & & & & & & 137 & 278 & & \\
\hline Rochester, NY & Roc & 603 & & & & & & & & & 214 & & \\
\hline Syracuse, NY & Syr & 676 & & & & & & & & & 141 & 231 & \\
\hline Albany, NY & Alb & 817 & & & & & & & & & & & 182 \\
\hline
\end{tabular}

Table 1: City pairs and corresponding distances along I-90 for trips between 100 and 300 miles ("Spr" and "Bos" stand for Springfield, MA and Boston, MA, respectively)

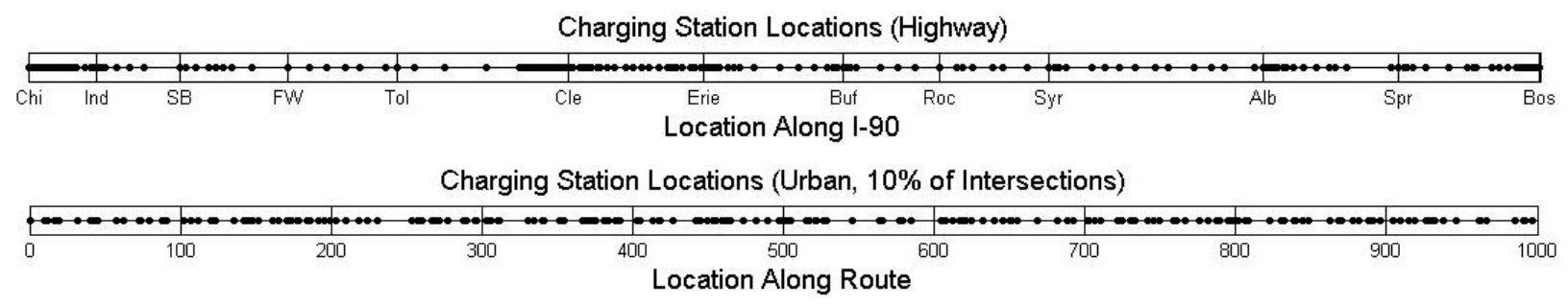

Figure 4: Charging station (node) locations along highway and urban routes

We compare the recharging policies of the three algorithms for both highway and urban driving, for all 22 
trips shown in Table 1, and for all combinations of the following parameter settings when the overcharging cost function is assumed to be of the form

$$
\begin{gathered}
f(x)=k_{1}\left(\exp \left\{x / k_{2}\right\}-1\right) . \\
q_{m a x}^{h w y} \in\{70,75,80,85,90,95,100\} ; q_{\max }^{u r b} \in\{100,105,110,115,120,125,130\} \\
\alpha \in\{0.75,0.76,0.77,0.78,0.79,0.80,0.81,0.82,0.83,0.84,0.85\} \\
k_{1} \in\{1.8,1.9,2.0,2.1,2.2\} \\
k_{2}^{h w y} \in\{4.8,4.9,5.0,5.1,5.2\} ; k_{2}^{u r b} \in \frac{q_{\max }^{u r b}}{q_{\max }^{u r b}-30} \cdot\{4.8,4.9,5.0,5.1,5.2\} \\
s \in\{1,2,3,5,10,25,50,75,100,150,200,250,300,400,500,600,800,1000\}
\end{gathered}
$$

The range of values for $q_{\max }$ corresponds to the maximum driving range of the 2014 Nissan Leaf under various weather and driving conditions (Fuel Economy Guide (2014)). Since the Leaf (and EVs in general) gets better mileage in an urban setting as opposed to on the highway due to regenerative braking and other factors, we use a lower range of values for highway driving $\left(q_{\text {max }}^{\text {hwy }}\right)$ than for urban driving $\left(q_{\text {max }}^{u r b}\right)$. Rather than use traditional units of kilowatt-hours, we assume that the energy consumed per mile traveled is constant and instead use the amount of energy required to travel one mile as our unit of measure for $q_{\max }$ (e.g., $q_{\max }=70$ implies that the vehicle can travel 70 miles on a full charge). For $\alpha$, the most commonly used value in practice is 0.8 (Nissan Leaf brochure (2014)), but we consider the interval $[0.75,0.85]$ to account for possible error. Equation (11) is based on the manner in which the charging current decreases during the constant-voltage phase of recharging (Dearborn (2006)), and the parameters $k_{1}$ and $k_{2}$ were calibrated based on one driver's experience with overcharging a Leaf (Laur (2013)). We distinguish $k_{2}^{h w y}$ from $k_{2}^{u r b}$ so that the maximum overcharging cost per stop is similar in both the highway and urban settings.

The parameters $q_{\max }, \alpha, k_{1}$, and $k_{2}$ are all dependent on the vehicle, but the stopping cost $s$ is primarily dependent on the driver. As a result, we include a wide range of values to capture different levels of aversion to stopping that the driver may have. The smallest values represent cases where the stopping cost consists only of the time required to access a charging station as well as a possible nominal fee charged by the station operator. The larger values capture cases in which the driver is more averse to stopping than to overcharging.

It is worth noting that the specific value assigned to $\gamma$ (the cost per unit of energy recharged) does not affect the structure of any of the policies, either optimal or heuristic. Each policy recharges exactly the 
same amount, namely $\sum_{i \in S_{P}} h_{i}$, and thus the total recharging cost of any policy is $\gamma \sum_{i \in S_{P}} h_{i}$, which is a constant. In the following analysis, we set $\gamma=4$ to correspond to charging rates at Level 2 stations (Nissan Leaf brochure (2014)). On a similar note, although one could argue that the first stopping cost in any trip should be disregarded since the driver could have recharged his or her vehicle the night prior and not incurred any inconvenience or delay by plugging in to recharge, this cost is the same regardless of the policy. Therefore, the structure of any policy for which the vehicle's initial charge level is zero does not change when the first stopping cost is included in the total cost. The structure of an optimal policy can vary for cases in which the vehicle's initial charge level is nonzero and greater than $h_{1}$, however. If the initial stopping cost is included in the total cost, then by Lemma 5 , the vehicle will not stop to recharge at the first node. On the other hand, if the first stopping cost is not included, then it may be optimal for the vehicle to recharge an additional amount at the origin before proceeding. This is especially true if the vehicle's initial charge level is below $\alpha q_{\max }$.

\subsection{Recharging policy cost analysis}

In this section, we analyze the performance of the two heuristic algorithms relative to each other. The solution quality of the recharging policies generated by both heuristics is illustrated in Figure 5. For small values of $s$, the policies generated by Heuristic 1 are optimal in the vast majority of instances, but the heuristic's performance deteriorates as the stopping cost increases. Heuristic 2, on the other hand, performs best when $s$ is high and not as well for smaller values. These patterns are to be expected since Heuristic 1's policies tend to include more stops than Heuristic 2's policies. (The same patterns also hold for trips longer than 300 miles. Additional figures illustrating the results are presented in Appendix A2.)

Comparing the highway and urban settings, Heuristic 2 performs closer to optimal over all values of $s$ along the urban route. However, the number of instances in which Heuristic 2 outperforms Heuristic 1 is lower for higher $s$ values despite Heuristic 1's cost ratio being slightly higher than in the highway setting. Heuristic 2 's improved performance can be attributed to the higher $q_{\max }$ values, which leads to fewer recharging stops per trip and thus fewer opportunities to overcharge. Although Heuristic 1 appears to perform a bit worse in the urban setting for higher values of $s$, its mean cost ratio is skewed towards instances in which the number of stops in the policy generated by Heuristic 1 is one more than the number of stops in an optimal policy. The ratio of the number of stops in a Heuristic 1 policy to that of an optimal policy can be as high as $2 / 1=2$ for the urban routes versus only $3 / 2=1.5$ for the highway routes due to the higher $q_{\max }$ values used in the urban setting. At the same time, the number of instances in which Heuristic 1 yields an optimal 

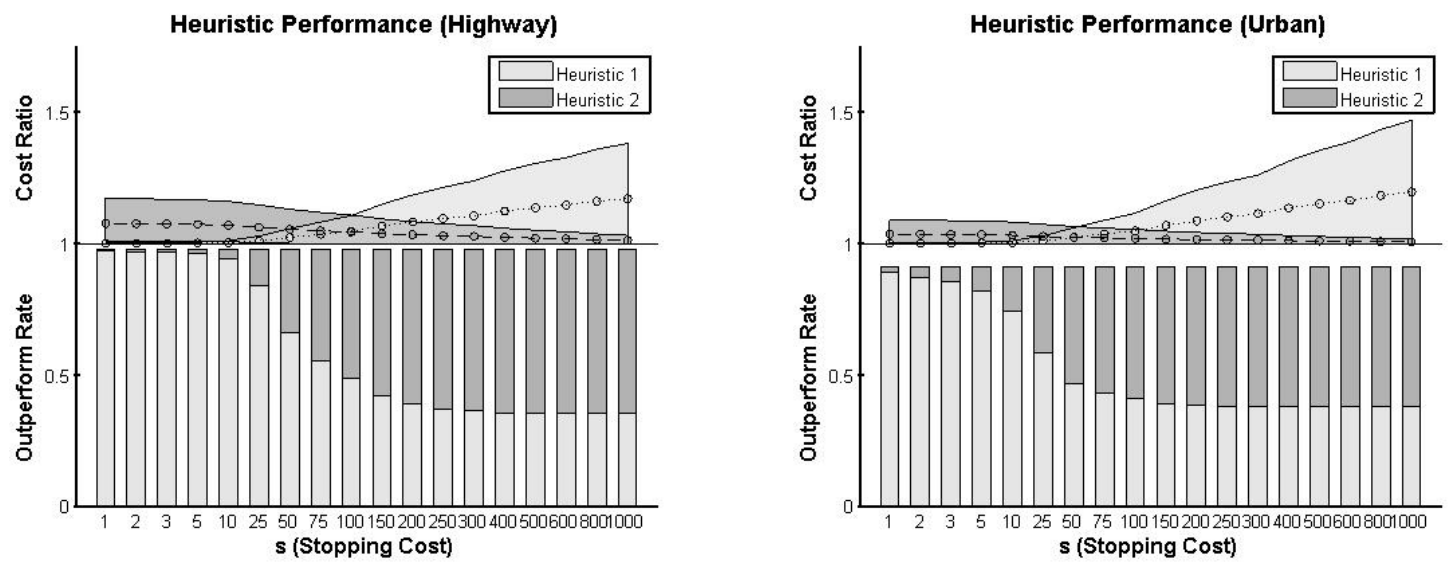

Figure 5: Heuristic recharging policy performance for different $s$ values in highway and urban settings; Outperform Rate measures the fraction of all instances (for all parameter settings and trips) in which the specified heuristic yields a policy with a lower cost than that of the other heuristic; and Cost Ratio is the cost of the heuristic's policy divided by the cost of an optimal policy (mean values with 10th-90th interpercentile ranges are shown)

policy is greater for the urban route.

The relative performance of the two heuristics in an urban setting is further illustrated in Figure 6 for different densities of charging stations. Overall, Heuristic 1 performs better as the density of charging stations increases, and the opposite is true for Heuristic 2. This observation can be attributed to the fact that a greater presence of charging stations allows the vehicle to recharge closer to $\alpha q_{\max }$ in Heuristic 1 policies and closer to $q_{\max }$ in Heuristic 2 policies. As a result, the number of stops in both heuristics' policies decreases on average, and thus the cost of Heuristic 1 policies also decreases. Heuristic 2 policies, on the other hand, experience greater overcharging costs to counteract the lower stopping costs, and the net effect is an increase in the total cost.

\subsection{Battery health considerations}

Battery degradation is important for EV drivers to understand due to the high replacement cost of batteries, but it is also a difficult metric to gauge since it is influenced by a number of different factors. Fortunately, our model captures several of the most significant factors, such as overcharging and number of charging cycles (measured by the number of recharging stops). In this section, we examine some of the metrics that cause battery degradation.

The average number of times that the vehicle stops to recharge under optimal and heuristic recharging policies over all parameter settings and trips (normalized by distance) for each route type is shown in Figure 

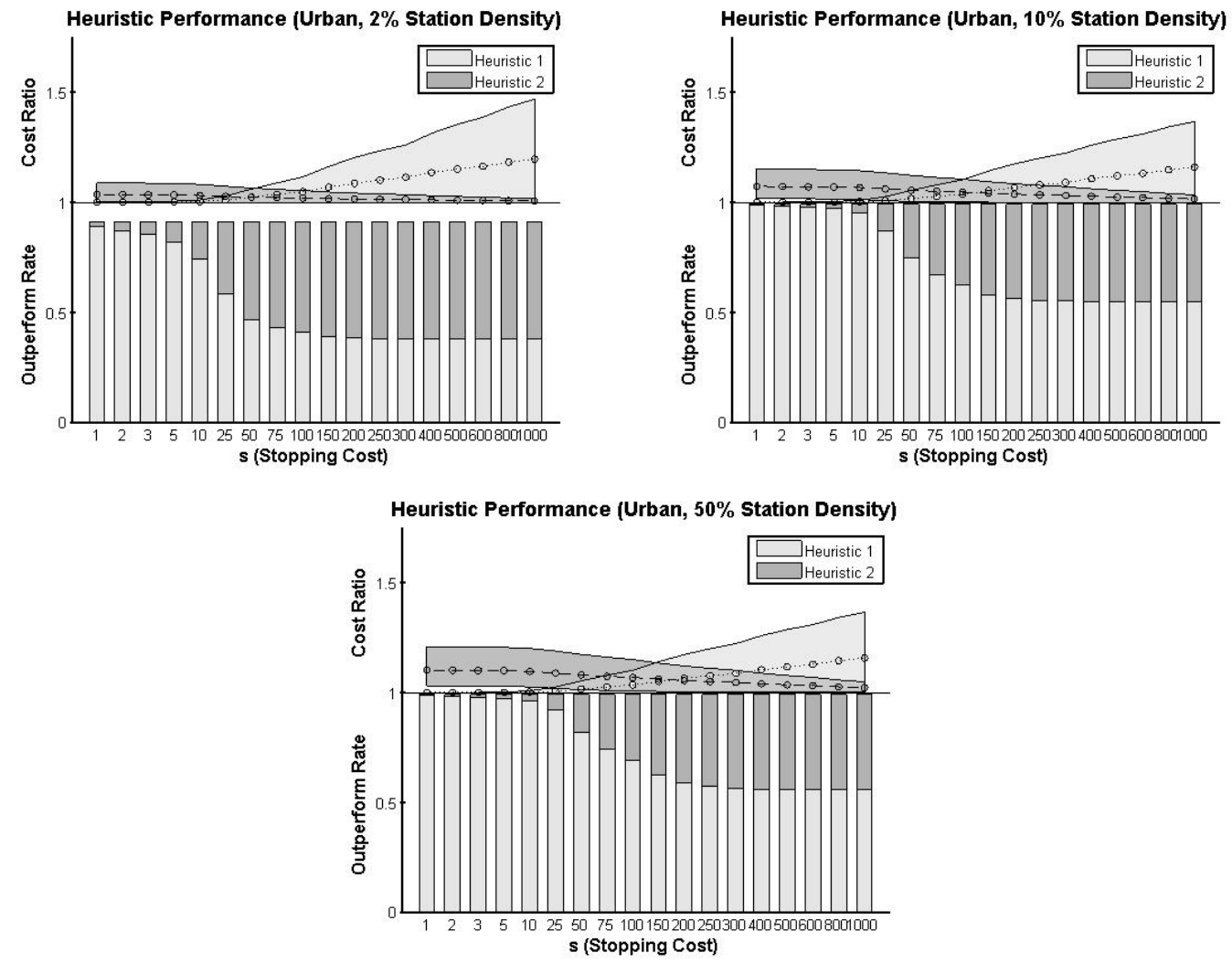

Figure 6: Heuristic recharging policy performance for urban routes with varying charging station densities (percentages of intersections with charging stations); mean Cost Ratios with 10th-90th interpercentile ranges are shown

7. The lower number of stops along the urban route relative to the highway route is due mainly to the larger values of $q_{\max }$. Because Heuristic 1 avoids overcharging, its policies have the greatest number of stops, and Heuristic 2 policies achieve the minimum number of stops. It can also be observed that relative to the number of stops in optimal recharging policies, the number of stops in Heuristic 2 policies is closer than that of Heuristic 1. This observation makes sense since Heuristic 2 policies can have the same number of stops as optimal policies despite possibly being suboptimal, whereas Heuristic 1 policies must have more stops than optimal policies whenever they are suboptimal.

Repeatedly recharging a battery causes degradation, but the extent to which the battery is recharged also affects the rate of degradation. Whereas Figure 7 shows the total average number of recharging stops for each trip, Figure 8 displays the average number of stops at which the vehicle overcharges (over all parameter settings and trips). Because no pair of consecutive nodes in either route is separated by more than $\alpha q_{\max }$ for any set of parameters, Heuristic 1 never requires overcharging. The difference of approximately 0.5 stops per 100 miles corresponds to an average of around one stop per trip that does not have overcharging. This 


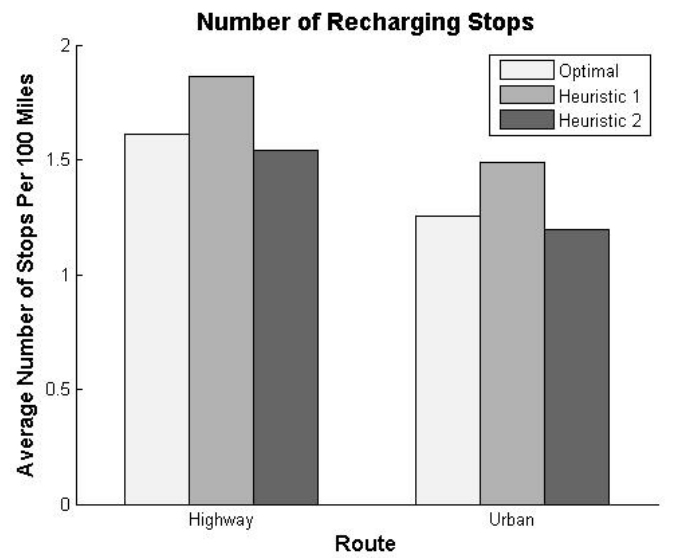

Figure 7: Number of stops in optimal and heuristic recharging policies, averaged over all parameter sets and trips (normalized by distance)

is usually the final stop in each trip since it can occur shortly before the final node in the segment. For optimal policies, by further examining Figures 7 and 8, approximately one-third of stops have overcharging, which implies that overcharging is slightly less favorable than including an extra recharging stop in terms of cost. This is especially true along the urban route, where it is much easier to have evenly spaced stops.

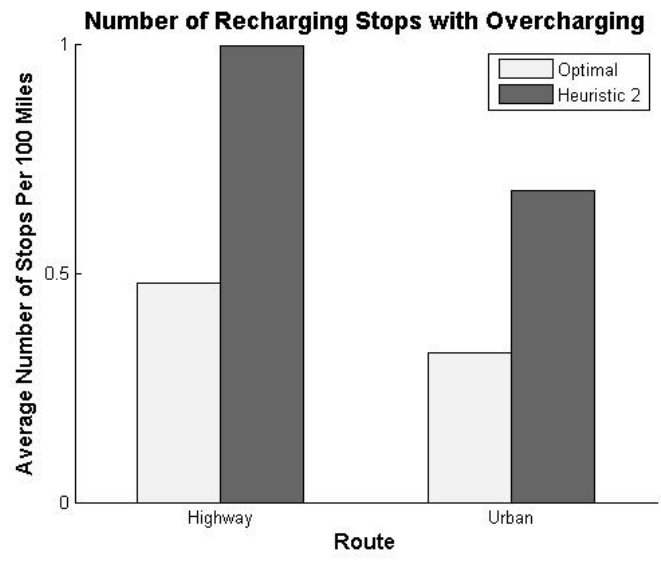

Figure 8: Number of stops with overcharging in optimal and Heuristic 2 recharging policies, averaged over all parameter sets and trips (normalized by distance)

In addition to the number of charging cycles, the total amount of energy overcharged by a battery affects its lifespan. The average fraction of recharging activity for each trip that involves overcharging is displayed in Figure 9. Again, because Heuristic 1 does not require overcharging, it is not shown in the figure. It can be observed that across all trips, optimal policies usually overcharge for less than $3 \%$ of all recharging activity while Heuristic 2 policies overcharge for between $8 \%$ and $12 \%$ of the total recharging amount. The difference 
is greater for the highway route, where optimal policies usually have less than one stop with overcharging and the irregular node spacing leads Heuristic 2 to overcharge more at each stop than along the urban route. While part of the difference can be attributed to the fewer number of stops with overcharging in optimal policies than in Heuristic 2 policies, a comparison of Figures 8 and 9 suggests that the amount overcharged per stop is less in optimal policies, which is to be expected since optimal policies only overcharge to avoid incurring an extra stopping cost.

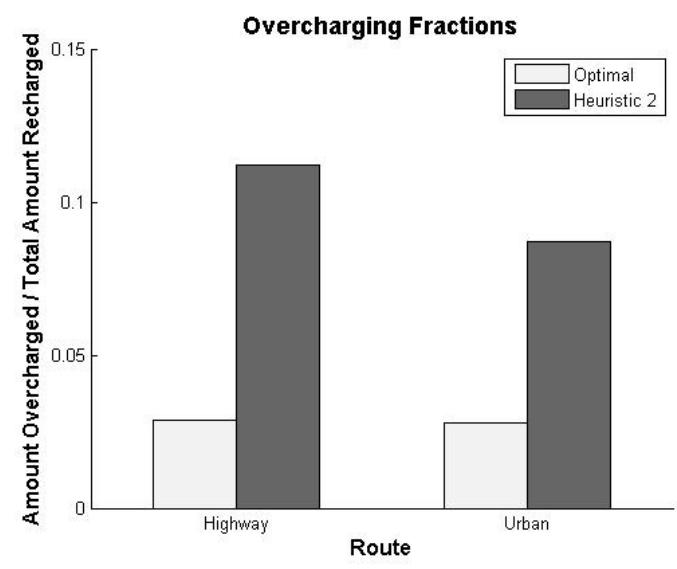

Figure 9: Fraction of all recharging activity for which overcharging costs are incurred in optimal and Heuristic 2 recharging policies, averaged over all parameter sets and trips

\subsection{Implementation of model extensions}

Based on our discussion in Section 5, we implement two of the extensions to our model: stochastic travel costs (see Section 5.2) and nonhomogeneous charging stations (see Section 5.3). We show how the two heuristics perform under the new settings and describe some modifications to adapt all three solution algorithms to each of the specialized cases.

\subsubsection{Stochastic travel costs}

If the travel $\operatorname{costs} h_{i}$ are stochastic, then Algorithm 1 no longer yields an optimal recharging policy. Nevertheless, we implement a version of the algorithm tailored for the case of stochastic travel costs and compare its performance to that of the two heuristics. We model uncertainty by adding an independent random error term $\varepsilon_{i}$ to each travel cost that is uniformly distributed on the interval $\left[-e h_{i},+e h_{i}\right]$ for a given uncertainty level $e>0$ (we consider $e \in\{0.2,0.5\}$ in our analysis). We also consider the values 4 and 100 for the penalty term $\beta$ that is incurred whenever the vehicle arrives at a node with a negative charge level. Setting $\beta=4$ 
yields a penalty that is equal to the charging cost rate $\gamma$ whereas having $\beta=100$ discourages policies that do not recharge a sufficient amount at each stop.

As mentioned in Section 5.2, the presence of uncertainty in the model makes it difficult to plan to arrive at each recharging stop with zero charge level, and the penalty for arriving with a negative charge level suggests that an optimal policy should attempt to maintain a safety buffer so that the likelihood of the vehicle's charge level becoming negative is reduced (see Tayur et al. (1999) and de Kok and Graves (2003) for some examples of buffer use in inventory models). We consider fixed safety buffer levels of $0 \%, 10 \%$, and $20 \%$ of $q_{\max }$ in our analysis. Note that in the deterministic setting, maintaining a fixed buffer is equivalent to shifting the cost function $(c(r, 0)$ becomes $c(r, b)$ for buffer level $b)$ and Algorithm 1 can still be used to obtain an optimal policy. Although a recharging policy optimized for the stochastic setting would likely have a buffer that varies along the path (i.e., becoming greater when the distance between planned recharging stops is greater), we do not consider such a sophisticated buffer strategy in this analysis. It would require information regarding the distributions of the $H_{i}$ variables as well as any correlations among them, and policies would need to be calculated at each node for the entire spectrum of possible charge levels, which would be computationally demanding.

To implement each of our solution methods when the travel costs are stochastic, we first obtain a policy for the deterministic setting when all error terms $\varepsilon_{i}$ are zero. The vehicle follows this policy, recharging at each stop an amount such that its charge level equals the buffer plus the expected charge required to reach the next stop, for as long as its charge level does not become negative prior to reaching a planned stop. In such a case, a new policy is obtained for the remainder of the path, again calculated using the deterministic setting. The vehicle then follows this revised policy for as long as its charge level remains non-negative. This process of following and updating the policy repeats until the vehicle reaches the end of the path. For each set of parameters, 10 different realizations of the stochastic path are simulated.

It can be seen in Figure 10 that varying the uncertainty level does not have much of an effect on the relative performance of the heuristics. (From here on, we examine only the highway routes since the urban routes exhibit similar patterns.) Increasing the uncertainty does increase the variability of each heuristic's performance, but the overall patterns remain the same. Varying the buffer level, however, has a much more noticeable impact (see Figure 11). When there is no buffer, all three algorithms have the potential to perform poorly, and in several instances, at least one of the heuristics outperforms the variation of Algorithm 1. (Recall that this algorithm no longer yields an optimal policy when travel costs are stochastic, so it is not guaranteed to perform the best among all three algorithms.) Heuristic 2 is the most resilient among the 
solution methods to uncertainty since it yields policies with the fewest recharging stops and thus the fewest opportunities to arrive at a node with a negative charge level, especially when the required buffer level is low. Increasing the buffer to $10 \%$ of $q_{\max }$ improves the performance of Heuristic 1, and increasing it further to $20 \%$ causes the relative performance of the heuristics to be similar to that in the deterministic setting.

When the buffer level is optimized for each solution method rather than fixed, the two heuristics offer less room for improvement over Algorithm 1. Figure 12 shows the results when the buffer level is optimized for each algorithm over all buffers between $0 \%$ and $30 \%$ in increments of $1 \%$. For each parameter setting, the best buffer for each heuristic method over all sampled paths is chosen, and the resulting mean costs are then divided by the mean cost of Algorithm 1 with its optimal buffer to obtain the cost ratios. The mean performance of each heuristic is never more than $0.5 \%$ lower than that of the modified Algorithm 1, and the 10th-percentile cost ratios are all above 0.96 . This suggests that even in a stochastic setting, the variation of Algorithm 1 performs well relative to the heuristics as long as the buffer level is optimized. Lastly, the effect of varying the penalty term is shown in Figure 13. When $\beta=\gamma$, the impact of uncertainty on the total cost of a policy is nullified, and the results appear similar to those of the deterministic case.

\subsubsection{Nonhomogeneous charging stations}

In our implementation up to this point, we have used the same recharging cost function at every node representing Level 2 charging stations. We now assume that Level 3 stations are present at every major city and have a recharging cost rate $\gamma^{\prime}$ such that $\gamma^{\prime}<\gamma$. We let the stopping costs and overcharging cost functions remain the same for all stations - both Level 2 and 3. As discussed in Section 5.3, we are able to obtain an optimal recharging policy in such a scenario with backward recursion. The performance of the heuristic algorithms relative to optimal policies is illustrated in Figure 14. Note that the heuristics do not take into account the locations of Level 3 stations and thus are not well equipped to take advantage of the lower recharging cost rate. This is best seen when the stopping cost is low since an optimal policy is more likely to stop at Level 3 stations even if it means having to make more stops overall. Increasing the frequency of Level 3 stations, as well as lowering the Level 3 recharging cost rate, both exacerbate this effect. As the stopping cost increases, however, the benefits of stopping to recharge more frequently in order to take advantage of Level 3 stations diminishes, and the gap between the optimal and heuristic policies becomes smaller.

We also tested two additional heuristics, labeled Heuristic 3 and Heuristic 4, that consider the locations of Level 3 charging stations and attempt to utilize them whenever possible. Whereas Heuristics 1 and 2 

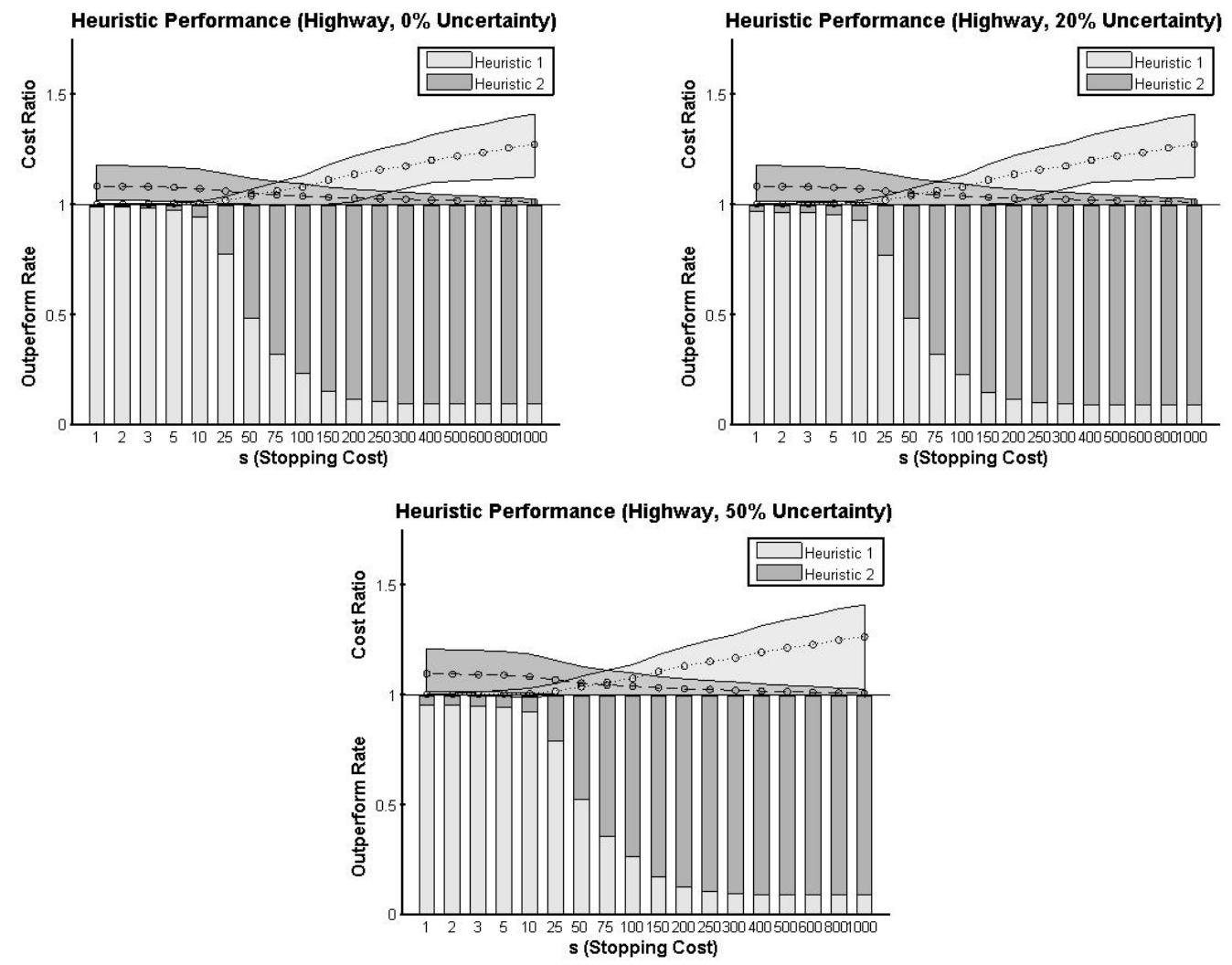

Figure 10: Heuristic recharging policy performance for highway routes in stochastic setting with varying uncertainty level $\left(b=0.2 q_{\max }, \beta=100\right)$; mean Cost Ratios with 10th-90th interpercentile ranges are shown

direct the vehicle to plan its next stop at the furthest station from its current stop location within $\alpha q_{\max }$ and $q_{\max }$, respectively, regardless of whether the charging station is Level 2 or 3; Heuristics 3 and 4 direct the vehicle to recharge at the furthest Level 3 station from its current stop location within $\alpha q_{\max }$ and $q_{\max }$, respectively. In the absence of any Level 3 stations within the allowable range of the vehicle, Heuristics 3 and 4 direct the vehicle to the furthest Level 2 station (i.e., they become identical to Heuristics 1 and 2). The performance of these two new heuristics is presented in Figure 15. Overall, Heuristic 4 performs quite well, especially in comparison to Heuristic 3. In fact, when Level 3 stations offer greater opportunities for cost savings (either with more stations or a lower recharging cost rate), the mean performance of Heuristic 4 is better than that of Heuristic 3 over all values of $s$. Furthermore, Heuristic 4 outperforms Heuristic 3 in the majority of instances. This is due in part to the fact that Heuristic 4 saves on both recharging and overcharging costs relative to Heuristic 2 (by recharging at Level 3 stations), yet Heuristic 3 incurs much greater stopping costs than Heuristic 1 does. The relative performance of Heuristics 1 and 2 to Heuristics 3 and 4 is further depicted in Figure 16. The tradeoff between the two sets of heuristics is most clearly seen 

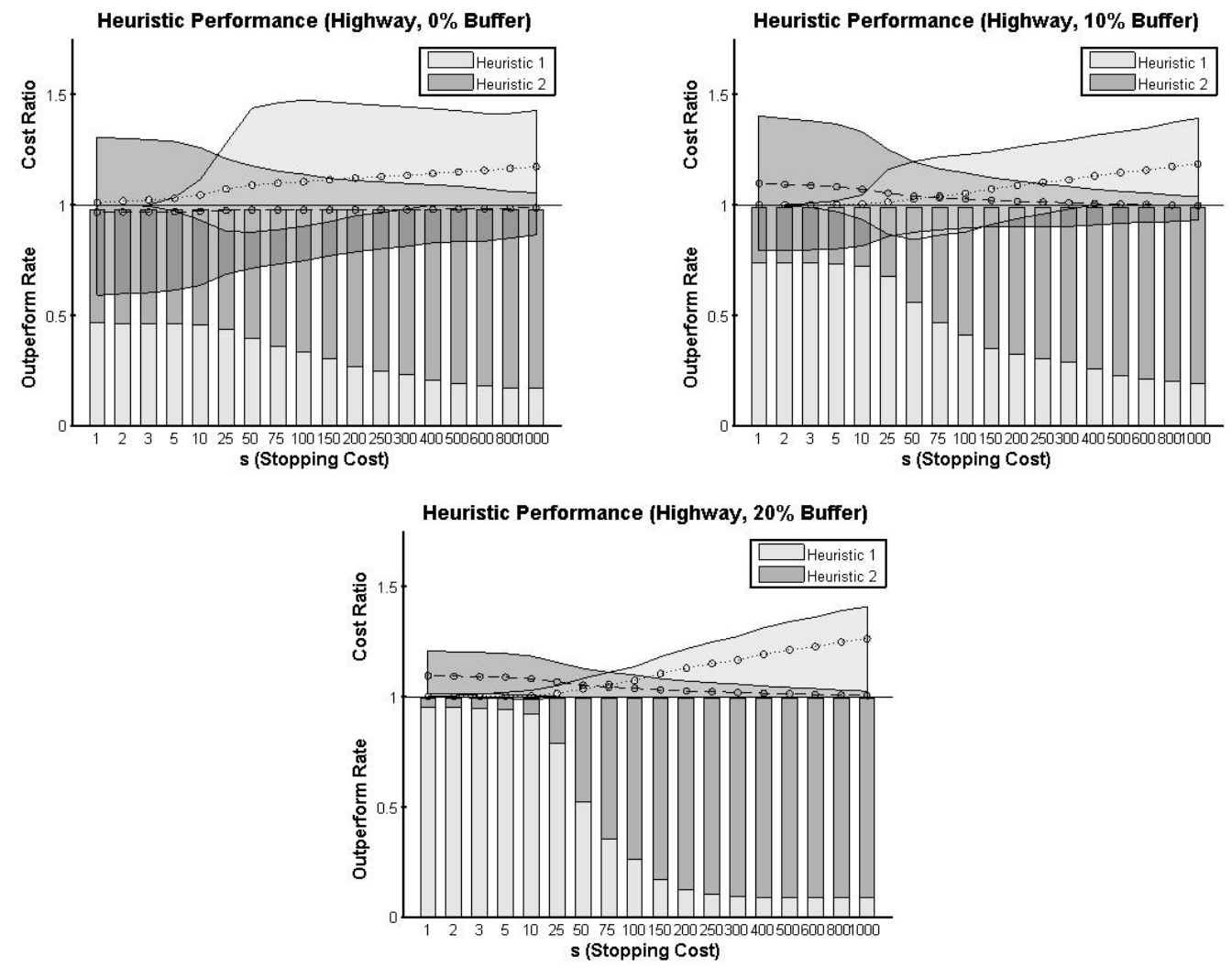

Figure 11: Heuristic recharging policy performance for highway route in stochastic setting with varying buffer level as percentage of $q_{\max }(e=0.5, \beta=100)$; mean Cost Ratios with 10th-90th interpercentile ranges are shown

in the case where Level 3 stations are more prevalent, with Heuristics 3 and 4 performing at their best when the stopping cost is low. For the other scenarios, however, neither set of heuristics outperforms the other in approximately $75 \%$ of instances.

\section{Conclusions and future work}

In this paper, we study the problem of finding an optimal recharging policy for an EV along a fixed path. We first determine properties of optimal policies, and based on those properties, we design methods for obtaining optimal recharging policies for general paths, paths with continuous charging capability, and paths with equidistant charging locations. We also develop two heuristics - one that minimizes overcharging costs (1) and another that minimizes the number of recharging stops (2) - and analyze the quality of their recharging policies, both theoretically and empirically for a designated stretch of U.S. Interstate 90 and a simulated urban setting. We find that the heuristics perform inversely to each other relative to the stopping 


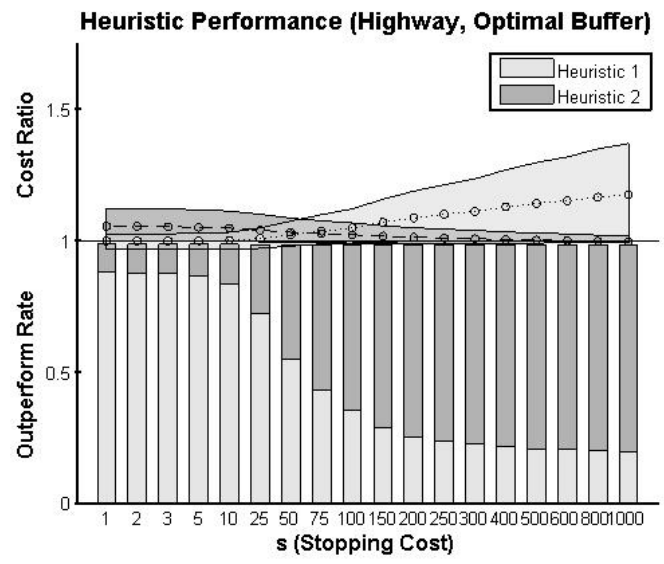

Figure 12: Heuristic recharging policy performance for highway route in stochastic setting with optimized buffer level (between $0 \%$ and 30\%, increments of 1\%); mean Cost Ratios with 10th-90th interpercentile ranges are shown
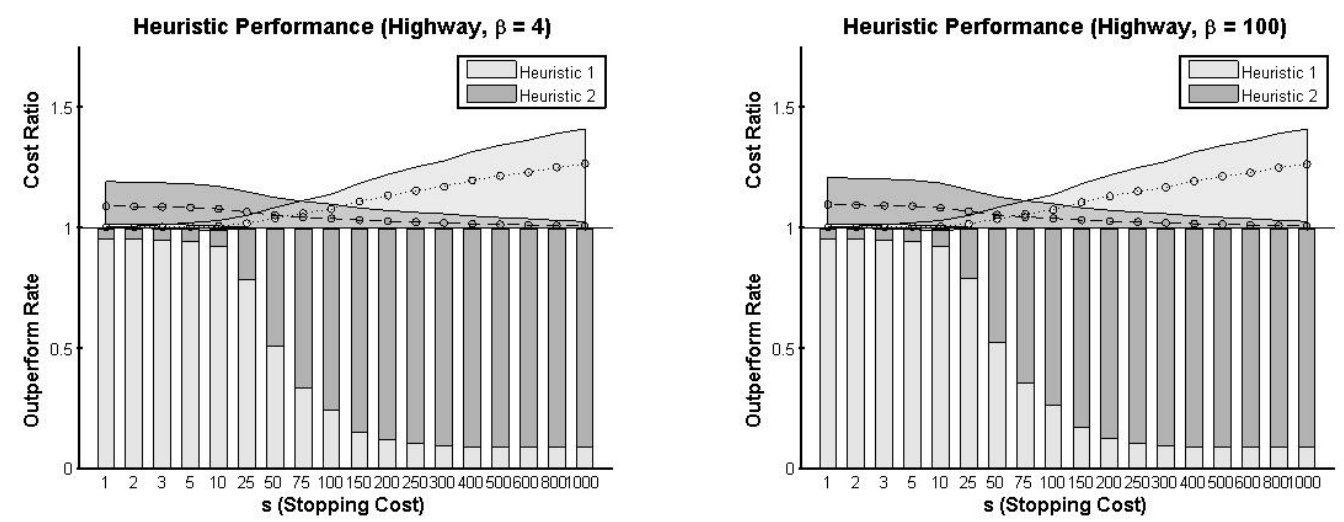

Figure 13: Heuristic recharging policy performance for highway route in stochastic setting with varying penalty term $\left(e=0.5, b=0.2 q_{\max }\right)$; mean Cost Ratios with 10th-90th interpercentile ranges are shown

cost and that the first heuristic performs best in a highway setting and when the number of nodes along the path is large whereas the second heuristic performs best in an urban setting when the nodes are more evenly spaced. Furthermore, we develop and discuss solution methods for several different extensions to our model.

An important aspect of our work is the inclusion of a realistic recharging model for electric vehicles. While most of the literature on vehicle refueling policies has focused primarily on the limited range of the vehicles, we show in this paper that the costs associated with battery overcharging can significantly influence recharging decisions, and thus, they should be taken into consideration when determining recharging policies for EVs. The simpler models of vehicle refueling used for conventional gasoline-powered vehicles are not suitable for EVs and require major enhancements before they can be used to improve our understanding of the various influences that battery dynamics have on EV recharging decisions. 

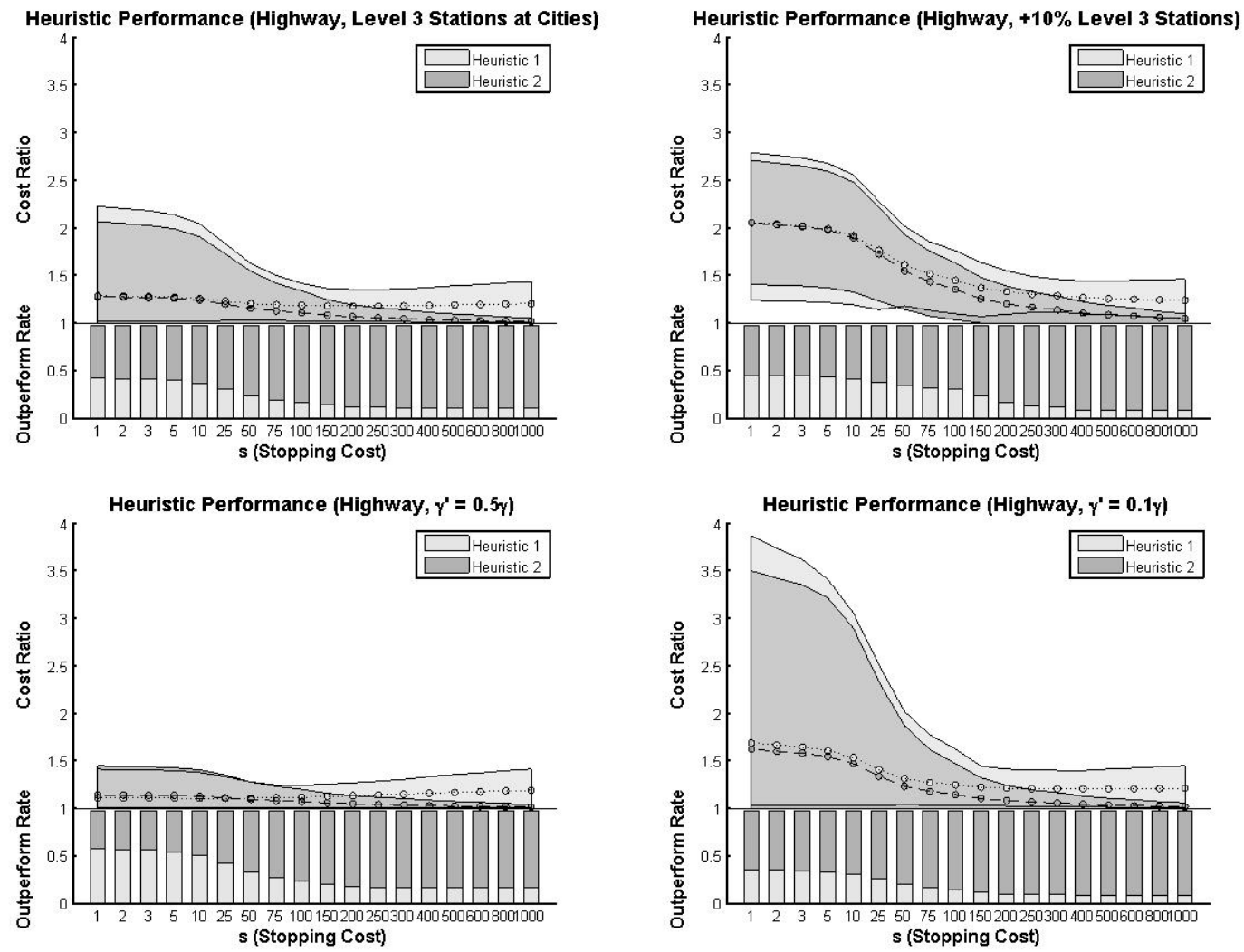

Figure 14: Heuristic 1 and 2 recharging policy performance for highway route with Level 2 and 3 charging stations (Row 1: $\gamma^{\prime}=0.25 \gamma$, Level 3 stations at (left) major cities only and (right) major cities plus an additional $10 \%$ of non-city nodes; Row 2: Level 3 stations at major cities only); mean Cost Ratios with 10th-90th interpercentile ranges are shown

Our current model considers recharging policies along a given route. A more detailed model would simultaneously find a path within a network, as well as a recharging policy for that path in order to minimize the total travel cost. In our future work, we plan to extend our current analysis and results to a network setting in order to design algorithms that also determine the best path for the vehicle to take. Furthermore, we intend to integrate additional features into our model that capture such aspects of EV recharging as the impacts of overcharging and temperature on battery storage capacity, the ability to recover energy via regenerative braking, and time-of-use energy prices.

We also plan to study versions of our model with stochastic features such as travel costs, which we began to explore in this paper. The uncertainty in travel costs can be due to factors such as traffic, weather, and road conditions, and the variability on each arc may be correlated with other arcs or vary with time. In addition, we will consider models in which the availability of charging stations is uncertain and may vary with time as well. 

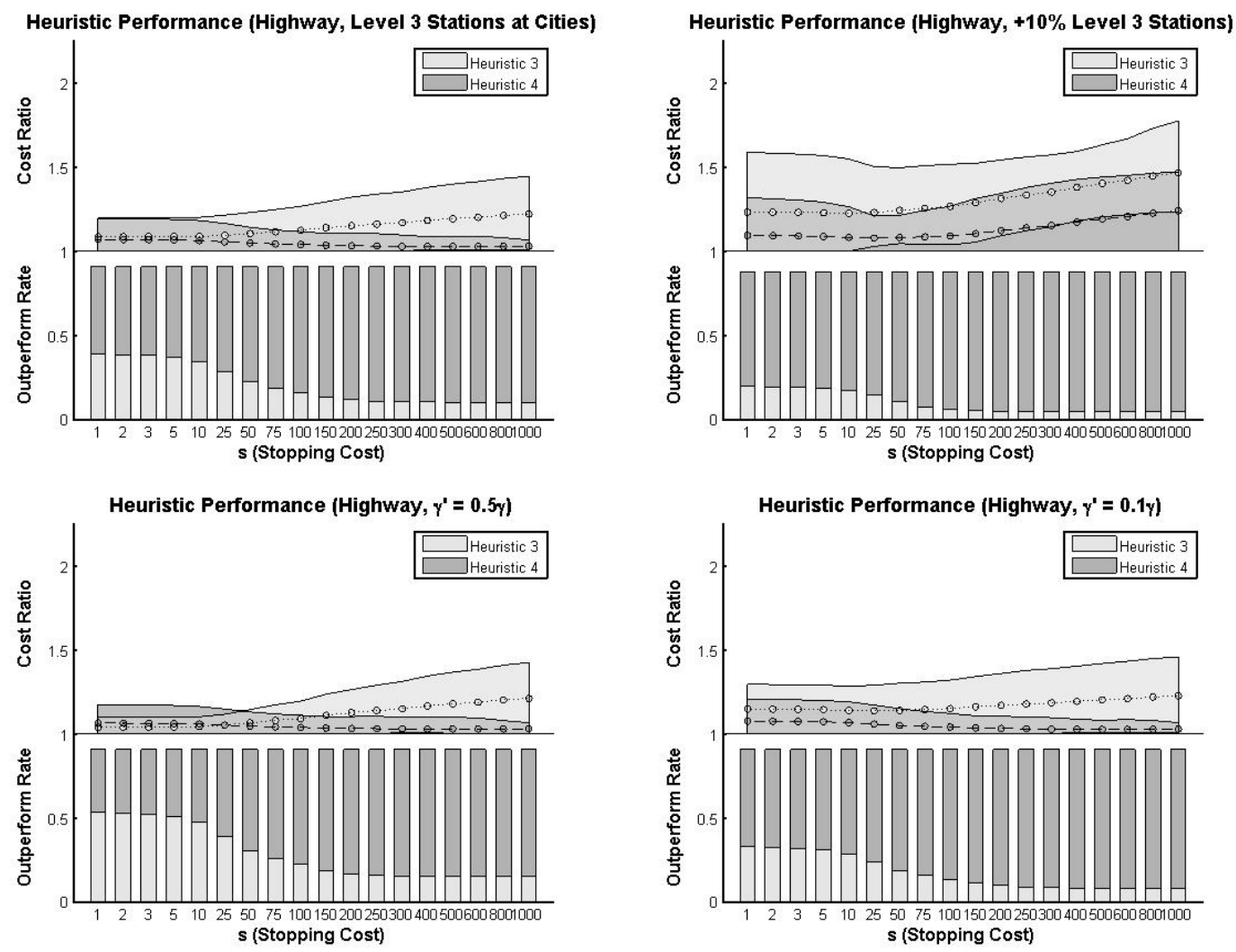

Figure 15: Heuristic 3 and 4 recharging policy performance for highway routes with Level 2 and 3 charging stations (Row 1: $\gamma^{\prime}=0.25 \gamma$, Level 3 stations at (left) major cities only and (right) major cities plus an additional $10 \%$ of non-city nodes; Row 2: Level 3 stations at major cities only); mean Cost Ratios with 10th-90th interpercentile ranges are shown

\section{Acknowledgement}

The authors would like to thank Scott Moura from the University of California, Berkeley for his insightful discussions. 

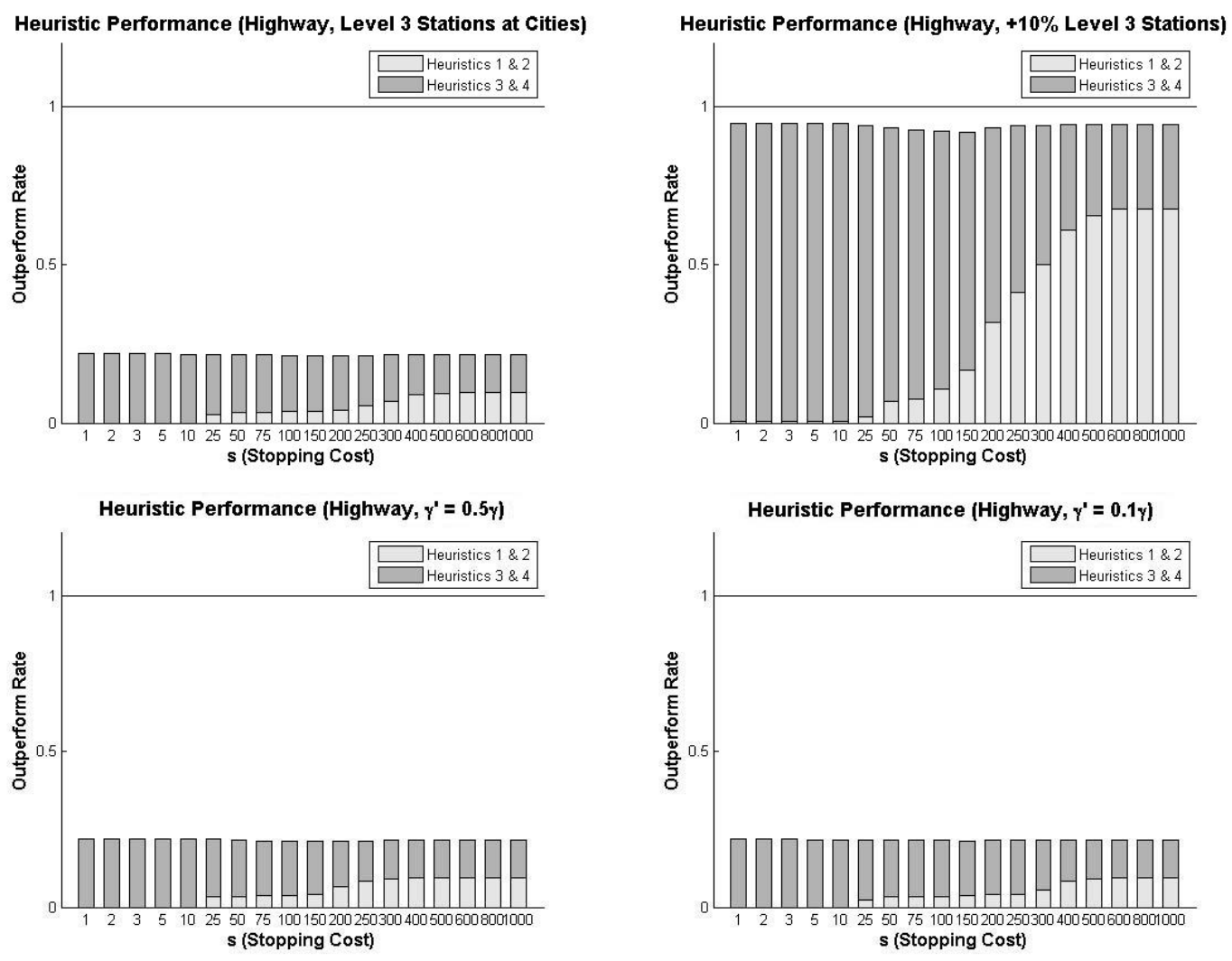

Figure 16: Heuristic recharging policy performance for highway route with Level 2 and 3 charging stations (Row 1: $\gamma^{\prime}=0.25 \gamma$; Row 2: Level 3 stations at major cities only); mean Cost Ratios with 10th-90th interpercentile ranges are shown

\section{References}

Artmeier, A., Haselmayr, J., Leucker, M., \& Sachenbacher, M. (2010). The shortest path problem revisited: Optimal routing for electric vehicles. Lecture Notes in Computer Science, 6359, 309-316.

Atamtürk, A., \& Küçükyavuz, S. (2005). Lot sizing with inventory bounds and fixed costs: Polyhedral study and computation. Operations Research, 53, 711-730.

Bellman, R., Glicksberg, I., \& Gross, O. (1955). On the optimal inventory equation. Management Science, 2 , 83-104.

Bhaskaran, S., Ramachandran, K., \& Semple, J. (2010). A dynamic inventory model with the right of refusal. Management Science, 56, 2265-2281.

Bulinskaya, E. V. (1967). Optimum inventory policies with a convex ordering cost function. Theory of Probability and Its Applications, 1, 9-21.

de Kok, A. G., \& Graves, S. C. (Eds.). (2003). Supply chain management: Design, coordination and operation (Vol. 11). Amsterdam: Elsevier. 
Dearborn, S. (2006). Develop affordable mixed-signal battery chargers. Electronic Design Europe. Retrieved from http://electronicdesign.com/energy/develop-affordable-mixed-signal-battery-chargers

Eisner, J., Funke, S., \& Storandt, S. (2011). Optimal route planning for electric vehicles in large networks. Proceedings of the 25th Association for the Advancement of Artificial Intelligence Conference.

Florian, M., Lenstra, J. K., \& Rinnooy Kan, A. H. G. (1980). Deterministic production planning: Algorithms and complexity. Management Science, 26, 669-679.

Fuel Economy Guide. (2014). U.S. Department of Energy, Office of Energy Efficiency and Renewable Energy, and U.S. Environmental Protection Agency. DOE/EE-0957. Retrieved from http://www.fueleconomy.gov/feg/pdfs/guides/FEG2014.pdf

Henig, M., Gerchak, Y., Ernst, R., \& Pyke, D. F. (1997). An inventory model embedded in designing a supply contract. Management Science, 43, 184-189.

Karlin, S. (1958). Optimal inventory policy for the Arrow-Harris-Marschak dynamic model. Studies in the Mathematical Theory of Inventory and Production, 135-154.

Khuller, S., Malekian, A., \& Mestre, J. (2007). To fill or not to fill: The gas station problem. Proceedings of the 15th Annual European Symposium on Algorithms, 4698, 534-545.

Klabjan, D., \& Sweda, T. (2011). The nascent industry of electric vehicles. Wiley Encyclopedia of Operations Research and Management Science.

Klampfl, E., Gusikhin, O., Theisen, K., Liu, Y., \& Giuli, T. J. (2008). Intelligent refueling advisory system. White paper.

Laur, D. (2013, April 10). Fast charging to 100\%. My Nissan Leaf Forum. Retrieved from http: //www . mynissanleaf . com/viewtopic $\cdot$ php?f=27\&t=12263\&start=10\#p282113

Lin, S. H., Gertsch, N., \& Russell, J. R. (2007). A linear-time algorithm for finding optimal vehicle refueling policies. Operations Research Letters, 35, 290-296.

Lin, S. H. (2008). Finding optimal refueling policies: A dynamic programming approach. Journal of Computing Sciences in Colleges, 23, 272-279.

Lin, S. H. (2008). Finding optimal refueling policies in transportation networks. Proceedings of the 4th International Conference on Algorithmic Aspects in Information and Management, 5034, 280-291.

Millner, A. (2010). Modeling lithium ion battery degradation in electric vehicles. Proceedings of the Conference on Innovative Technologies for an Efficient and Reliable Electricity Supply, 349-356.

Nissan Leaf Digital Brochure. (2014). Nissan USA. Retrieved from http://www.nissanusa.com/content/dam/nissan/ request-brochure/en/2014/pdf/2014-nissan-leaf-en.pdf 
Sachenbacher, M., Leucker, M., Artmeier, A., \& Haselmayr, J. (2011). Efficient energy-optimal routing for electric vehicles. Proceedings of the 25th Association for the Advancement of Artificial Intelligence Conference.

Serrao, L., Onori, S., Sciarretta, A., Guezennec, Y., \& Rizzoni, G. (2011). Optimal energy management of hybrid electric vehicles including battery aging. Proceedings of American Control Conference, 2125-2130.

Suzuki, Y. (2008). A generic model of motor-carrier fuel optimization. Naval Research Logistics, 55, 737-746.

Suzuki, Y. (2009). A decision support system of dynamic vehicle refueling. Decision Support Systems, 46, 522-531.

Sweda, T. M., \& Klabjan, D. (2012). Finding minimum-cost paths for electric vehicles. Proceedings of the 1st IEEE International Electric Vehicle Conference, 1-4.

Tayur, S., Ganeshan, R., \& Magazine, M. (Eds.). (1999). Quantitative models for supply chain management (Vol. 17). Springer Science \& Business Media. 


\section{Appendix A1: Supplemental proofs}

\section{Proof of Lemma 1}

Note that $\pi_{P}$ is feasible because $r_{i}=\sum_{j=i}^{m-1} h_{j}$ ensures that the vehicle will reach node $m$ (albeit with 0 charge remaining), $r_{m}=r_{m}^{*}+\left(r_{i}^{*}-\sum_{j=i}^{m-1} h_{j}\right)$ ensures that the vehicle departs node $m$ with the same charge level as it would have in $\pi_{P}^{*}$, and all other recharging amounts are the same as in $\pi_{P}^{*}$. We have

$$
\begin{aligned}
C\left(\pi_{P}^{*}\right)-C\left(\pi_{P}\right)= & \sum_{\ell=1}^{n}\left[c\left(r_{\ell}^{*}, \sum_{j=1}^{\ell-1}\left(r_{j}^{*}-h_{j}\right)\right)-c\left(r_{\ell}, \sum_{j=1}^{\ell-1}\left(r_{j}-h_{j}\right)\right)\right] \\
= & c\left(r_{i}^{*}, \sum_{j=1}^{i-1}\left(r_{j}^{*}-h_{j}\right)\right)-c\left(r_{i}, \sum_{j=1}^{i-1}\left(r_{j}-h_{j}\right)\right)+c\left(r_{m}^{*}, \sum_{j=1}^{m-1}\left(r_{j}^{*}-h_{j}\right)\right) \\
& -c\left(r_{m}, \sum_{j=1}^{m-1}\left(r_{j}-h_{j}\right)\right),
\end{aligned}
$$

since $r_{\ell}^{*}=r_{\ell}$ and $\sum_{j=1}^{\ell-1}\left(r_{j}^{*}-h_{j}\right)=\sum_{j=1}^{\ell-1}\left(r_{j}-h_{j}\right)$ for all $\ell \in\{1, \ldots, i-1, m+1, \ldots, n\}$ and

$$
c\left(r_{\ell}^{*}, \sum_{j=1}^{\ell-1}\left(r_{j}^{*}-h_{j}\right)\right)=c\left(r_{\ell}, \sum_{j=1}^{\ell-1}\left(r_{j}-h_{j}\right)\right)=0
$$

for all $\ell \in\{i+1, \ldots, m-1\}$ due to $r_{\ell}^{*}=r_{\ell}=0$ and $c(0, \cdot)=0$. We also have

$$
\sum_{j=1}^{i-1}\left(r_{j}^{*}-h_{j}\right)=\sum_{j=1}^{i-1}\left(r_{j}-h_{j}\right)=\sum_{j=1}^{m-1}\left(r_{j}-h_{j}\right)=0
$$

by definition of $i$ and $m$. It follows that

$$
\begin{aligned}
C\left(\pi_{P}^{*}\right)-C\left(\pi_{P}\right)= & c\left(r_{i}^{*}, 0\right)-c\left(r_{i}, 0\right)+c\left(r_{m}^{*}, r_{i}^{*}-\sum_{j=i}^{m-1} h_{j}\right)-c\left(r_{m}, 0\right) \\
= & \left(s+\gamma r_{i}^{*}+f\left(\left(r_{i}^{*}-\alpha q_{\max }\right)^{+}\right)\right)-\left(s+\gamma r_{i}+f\left(\left(\sum_{j=i}^{m-1} h_{j}-\alpha q_{\max }\right)^{+}\right)\right) \\
& +\left(s+\gamma r_{m}^{*}+\left[f\left(\left(r_{i}^{*}-\sum_{j=i}^{m-1} h_{j}+r_{m}^{*}-\alpha q_{\max }\right)^{+}\right)\right.\right. \\
& \left.\left.-f\left(\left(r_{i}^{*}-\sum_{j=i}^{m-1} h_{j}-\alpha q_{\max }\right)^{+}\right)\right]\right)
\end{aligned}
$$




$$
\begin{aligned}
& -\left(s+\gamma r_{m}+f\left(\left(r_{m}^{*}+r_{i}^{*}-\sum_{j=i}^{m-1} h_{j}-\alpha q_{\max }\right)\right)\right. \\
= & f\left(\left(r_{i}^{*}-\alpha q_{\max }\right)^{+}\right)-f\left(\left(\sum_{j=i}^{m-1} h_{j}-\alpha q_{\max }\right)^{+}\right)-f\left(\left(r_{i}^{*}-\sum_{j=i}^{m-1} h_{j}-\alpha q_{\max }\right)^{+}\right) \\
\geq & f\left(\left(r_{i}^{*}-\alpha q_{\max }\right)^{+}\right)-f\left(\left(\sum_{j=i}^{m-1} h_{j}-\alpha q_{\max }\right)^{+}+\left(r_{i}^{*}-\sum_{j=i}^{m-1} h_{j}-\alpha q_{\max }\right)^{+}\right) \\
\geq & f\left(\left(r_{i}^{*}-\alpha q_{\max }\right)^{+}\right)-f\left(\left(r_{i}^{*}-\alpha q_{\max }\right)^{+}\right) \\
= & 0,
\end{aligned}
$$

where (12) is true since both $\sum_{j=i}^{m-1} h_{j}>0$ and $r_{i}^{*}-\sum_{j=i}^{m-1} h_{j}>0$, and (13) is true since $f(\cdot)$ is convex and increasing. Because $\pi_{P}^{*}$ is an optimal policy, $C\left(\pi_{P}^{*}\right)=C\left(\pi_{P}\right)$ and $\pi_{P}$ is also an optimal policy.

\section{Proof of Lemma 2}

Suppose $r_{0}^{\prime}$ and $r_{1}^{\prime}$ are the amounts to recharge at nodes 0 and 1 , respectively, specified by policy $\pi_{P^{\prime}}^{*}$. Let $\pi_{P}=\left[r_{1}, \ldots, r_{n}\right]$ denote the recharging policy for $P$ that satisfies

$$
r_{\ell}= \begin{cases}r_{0}^{\prime}+r_{1}^{\prime}-h_{0}, & \ell=1 \\ r_{\ell}^{\prime}, & \ell \in S_{P} \backslash\{1\} .\end{cases}
$$

This policy is feasible because $r_{1}=r_{0}^{\prime}+r_{1}^{\prime}-h_{0}$ ensures that the vehicle departs node 1 with the same charge level as in $\pi_{P^{\prime}}^{*}$, and all other recharging amounts are the same as in $\pi_{P^{\prime}}^{*}$ (with the exception of node 0, which is not part of $P$ and therefore has no corresponding recharging amount in $\pi_{P}$ ). Then the total cost of $\pi_{P^{\prime}}^{*}$ exceeds that of $\pi_{P}$ by

$$
\begin{aligned}
C\left(\pi_{P^{\prime}}^{*}\right)-C\left(\pi_{P}\right)= & c\left(r_{0}^{\prime}, 0\right)+c\left(r_{1}^{\prime}, r_{0}^{\prime}-h_{0}\right)-c\left(r_{0}^{\prime}+r_{1}^{\prime}-h_{0}, 0\right) \\
= & {\left[s+\gamma r_{0}^{\prime}+f\left(\left(r_{0}^{\prime}-\alpha q_{\max }\right)^{+}\right)\right]+\left[s I_{\left\{r_{1}^{\prime}>0\right\}}+\gamma r_{1}^{\prime}+f\left(\left(r_{0}^{\prime}-h_{0}+r_{1}^{\prime}-\alpha q_{\max }\right)^{+}\right)\right.} \\
& \left.-f\left(\left(r_{0}^{\prime}-h_{0}-\alpha q_{\max }\right)^{+}\right)\right]-\left[s+\gamma\left(r_{0}^{\prime}+r_{1}^{\prime}-h_{0}\right)+f\left(\left(r_{0}^{\prime}+r_{1}^{\prime}-h_{0}-\alpha q_{\max }\right)^{+}\right)\right] \\
= & s I_{\left\{r_{1}^{\prime}>0\right\}}+\gamma h_{0}+f\left(\left(r_{0}^{\prime}-\alpha q_{\max }\right)^{+}\right)-f\left(\left(r_{0}^{\prime}-h_{0}-\alpha q_{\max }\right)^{+}\right) \\
\geq & \gamma h_{0}
\end{aligned}
$$


because $f(\cdot)$ is increasing. Since $\pi_{P}^{*}$ is an optimal recharging policy for $P$, it follows that

$$
C\left(\pi_{P^{\prime}}^{*}\right) \geq C\left(\pi_{P}\right)+\gamma h_{0} \geq C\left(\pi_{P}^{*}\right)+\gamma h_{0},
$$

as desired.

\section{Proof of Lemma 3}

Note that $\pi_{P}$ is feasible since $r_{i}=r_{i}^{*}+h_{m}$ ensures that the vehicle departs node $i$ with just enough charge to reach node $m+1$ (and $r_{i} \leq \alpha q_{\max }$ by supposition), and $r_{m+1}=r_{m}^{*}+r_{m+1}^{*}-h_{m}$ ensures that the vehicle departs node $m+1$ with the same charge level as in $\pi_{P}^{*}$. We define the following subpaths of $P$ along with corresponding recharging policies:

$$
\begin{aligned}
& P_{1}=(1, \ldots, m), \pi_{P_{1}}^{*}=\left[r_{1}^{*}, \ldots, r_{m-1}^{*}\right] \\
& P_{2}=(m, \ldots, n+1), \pi_{P_{2}}^{*}=\left[r_{m}^{*}, \ldots, r_{n}^{*}\right] \\
& P_{1}^{\prime}=(1, \ldots, m+1), \pi_{P_{1}^{\prime}}=\left[r_{1}, \ldots, r_{m}\right] \\
& P_{2}^{\prime}=(m+1, \ldots, n+1), \pi_{P_{2}^{\prime}}=\left[r_{m+1}, \ldots, r_{n}\right]
\end{aligned}
$$

where $\pi_{P_{1}}^{*}$ and $\pi_{P_{2}}^{*}$ are subpolicies of $\pi_{P}^{*}$, and $\pi_{P_{1}^{\prime}}$ and $\pi_{P_{2}^{\prime}}$ are subpolicies of $\pi_{P}$. These policies are all feasible due to Property 1, which ensures that the recharging policies can be subdivided at any node where the vehicle stops to recharge since the charge level there will be 0 . Then we have

$$
\begin{aligned}
C\left(\pi_{P}^{*}\right)-C\left(\pi_{P}\right) & =\left(C\left(\pi_{P_{1}}^{*}\right)+C\left(\pi_{P_{2}}^{*}\right)\right)-\left(C\left(\pi_{P_{1}^{\prime}}\right)+C\left(\pi_{P_{2}^{\prime}}\right)\right) \\
& \geq C\left(\pi_{P_{1}}^{*}\right)-C\left(\pi_{P_{1}^{\prime}}\right)+\gamma h_{m} \quad(\text { by Lemma } 2) \\
& =c\left(r_{i}^{*}, 0\right)-c\left(r_{i}^{*}+h_{m}, 0\right)+\gamma h_{m} \\
& =-\gamma h_{m}+f\left(\left(r_{i}^{*}-\alpha q_{\max }\right)^{+}\right)-f\left(\left(r_{i}^{*}+h_{m}-\alpha q_{\max }\right)^{+}\right)+\gamma h_{m} \\
& =0
\end{aligned}
$$

since $r_{i}^{*}+h_{m} \leq \alpha q_{\max }$. Because $\pi_{P}^{*}$ is an optimal policy, $C\left(\pi_{P}^{*}\right)=C\left(\pi_{P}\right)$ and $\pi_{P}$ is also an optimal policy. 


\section{Proof of Lemma 4}

Let $\mu_{i}$ denote the index of the $i^{\text {th }}$ recharging stop where $\hat{r}_{\mu_{i}}>0$ for all $i \in\{1, \ldots, \hat{\xi}\}$. Due to the construction of $\hat{\pi}_{P}$, it must be true that $\alpha q_{\max }<\hat{r}_{\mu_{i}}+\hat{r}_{\mu_{i+1}}$ for any $i \in\{1, \ldots, \hat{\xi}-1\}$. Then we have

$$
(\hat{\xi}-1) \alpha q_{\max }<\sum_{i=1}^{\hat{\xi}-1}\left(\hat{r}_{\mu_{i}}+\hat{r}_{\mu_{i+1}}\right)<2 \sum_{i=1}^{\hat{\xi}} \hat{r}_{\mu_{i}}=2 H
$$

which implies

$$
\hat{\xi}-1<\frac{2 H}{\alpha q_{\max }}
$$

and

$$
\hat{\xi} \leq\left\lceil\frac{2 H}{\alpha q_{\max }}\right\rceil
$$

In the extreme case where $h_{i}=\frac{1}{2} \alpha q_{\max }+\varepsilon(\varepsilon>0)$ for all $i \in S_{P}$, the above bound is tight since

$$
\hat{\xi}=\frac{H}{\frac{1}{2} \alpha q_{\max }+\varepsilon}<\lim _{\varepsilon \rightarrow 0}\left(\frac{H}{\frac{1}{2} \alpha q_{\max }+\varepsilon}\right)=\frac{2 H}{\alpha q_{\max }} .
$$

We thus have

$$
\hat{\xi} \leq\left\lfloor\frac{2 H}{\alpha q_{\max }}\right\rfloor
$$

which is also equal to $\left\lceil 2 H / \alpha q_{\max }\right\rceil$ if $2 H / \alpha q_{\max }$ is an integer, and equality holds when

$$
\varepsilon=\frac{H}{\left\lfloor 2 H / \alpha q_{\max }\right\rfloor}-\frac{1}{2} \alpha q_{\max } .
$$

Therefore, the lemma statement is valid.

\section{Proof of Lemma 5}

Note that $\pi_{P, q_{1}}$ is feasible because the vehicle can reach node $m$ without recharging, $r_{m}=r_{i}^{*}$ ensures that the vehicle departs node $m$ with the same charge level as it would have in $\pi_{P, q_{1}}^{*}$, and all other recharging amounts are the same as in $\pi_{P, q_{1}}^{*}$. We have

$C\left(\pi_{P, q_{1}}^{*}\right)-C\left(\pi_{P, q_{1}}\right)=\sum_{\ell=1}^{n}\left[c\left(r_{\ell}^{*}, q_{1}+\sum_{j=1}^{\ell-1}\left(r_{j}^{*}-h_{j}\right)\right)-c\left(r_{\ell}, q_{1}+\sum_{j=1}^{\ell-1}\left(r_{j}-h_{j}\right)\right)\right]$ 


$$
\begin{aligned}
= & c\left(r_{i}^{*}, q_{1}-\sum_{j=1}^{i-1} h_{j}\right)-c\left(r_{i}^{*}, q_{1}-\sum_{j=1}^{m-1} h_{j}\right) \\
= & \left(s+\gamma r_{i}^{*}+f\left(\left(q_{1}-\sum_{j=1}^{i-1} h_{j}+r_{i}^{*}-\alpha q_{\max }\right)^{+}\right)-f\left(\left(q_{1}-\sum_{j=1}^{i-1} h_{j}-\alpha q_{\text {max }}\right)\right)\right)- \\
& \left(s+\gamma r_{i}^{*}+f\left(\left(q_{1}-\sum_{j=1}^{m-1} h_{j}+r_{i}^{*}-\alpha q_{\max }\right)^{+}\right)-f\left(\left(q_{1}-\sum_{j=1}^{m-1} h_{j}-\alpha q_{\text {max }}\right)^{+}\right)\right) \\
\geq & \left.\nabla f\left(\left(q_{1}-\sum_{j=1}^{i-1} h_{j}+r_{i}^{*}-\alpha q_{\text {max }}\right)^{+}\right) \sum_{j=i}^{m-1} h_{j}-\nabla f\left(\left(q_{1}-\sum_{j=1}^{m-1} h_{j}-\alpha q_{\text {max }}\right)\right)^{+}\right) \sum_{j=i}^{m-1} h_{j}
\end{aligned}
$$

(by convexity of $f(\cdot)$ )

$$
\geq 0
$$

since $i<m, r_{i}^{*}>0$, and $f(\cdot)$ is increasing. Because $\pi_{P, q_{1}}^{*}$ is an optimal policy, $C\left(\pi_{P, q_{1}}^{*}\right)=C\left(\pi_{P, q_{1}}\right)$ and $\pi_{P, q_{1}}$ is also an optimal policy.

\section{Proof of Theorem 1}

Suppose for some continuous recharging policy $\rho_{P, \xi}=\left(\left[\lambda_{1}, \ldots, \lambda_{\xi}\right],\left[r_{1}, \ldots, r_{\xi}\right]\right)$ that $\sum_{\ell=1}^{i} r_{\ell}=P\left(\lambda_{i+1}\right)=$ $H \lambda_{i+1}$ for all $i \in\{1, \ldots, \xi\}$. Note that this condition is the continuous analog of Property 1 and there exists an optimal recharging policy that satisfies it. Also suppose that $r_{i}<r_{j}$ for some $i, j \in\{1, \ldots, \xi\}$. Let $\rho_{P, \xi}^{\prime}=\left(\left[\lambda_{1}^{\prime}, \ldots, \lambda_{\xi}^{\prime}\right],\left[r_{1}^{\prime}, \ldots, r_{\xi}^{\prime}\right]\right)$ be a policy with

$$
r_{\ell}^{\prime}= \begin{cases}r_{i}+\varepsilon, & \ell=i \\ r_{j}-\varepsilon, & \ell=j \\ r_{\ell}, & \ell \in\{1, \ldots, \xi\} \backslash\{i, j\}\end{cases}
$$

for some $\varepsilon \in\left(0, r_{j}-r_{i}\right)$ and $\sum_{j=1}^{i} r_{j}^{\prime}=P\left(\lambda_{i+1}^{\prime}\right)=H \lambda_{i+1}^{\prime}$ for all $i \in\{1, \ldots, \xi\}$. That is, the locations of recharging stops $i$ through $j$ (or $j$ through $i$ ) are adjusted such that $\sum_{\ell=1}^{i} r_{\ell}^{\prime}=P\left(\lambda_{i+1}\right)$ for all $i \in\{1, \ldots, \xi\}$. Note that this policy is feasible because the total amount recharged along the path is still $H$ and the vehicle only recharges when its charge level is 0 . Then we have

$$
D\left(\rho_{P, \xi}^{\prime}\right)-D\left(\rho_{P, \xi}\right)=\sum_{\ell=1}^{\xi}\left(c\left(r_{\ell}^{\prime}, 0\right)-c\left(r_{\ell}, 0\right)\right)
$$




$$
\begin{aligned}
= & c\left(r_{i}+\varepsilon, 0\right)-c\left(r_{i}, 0\right)+c\left(r_{j}-\varepsilon, 0\right)-c\left(r_{j}, 0\right) \\
= & {\left[s+\gamma\left(r_{i}+\varepsilon\right)+f\left(\left(r_{i}+\varepsilon-\alpha q_{\max }\right)^{+}\right)\right]-\left[s+\gamma r_{i}+f\left(\left(r_{i}-\alpha q_{\max }\right)^{+}\right)\right] } \\
& +\left[s+\gamma\left(r_{j}-\varepsilon\right)+f\left(\left(r_{j}-\varepsilon-\alpha q_{\max }\right)^{+}\right)\right]-\left[s+\gamma r_{j}+f\left(\left(r_{j}-\alpha q_{\max }\right)^{+}\right)\right] \\
= & \left(f\left(\left(r_{i}+\varepsilon-\alpha q_{\max }\right)^{+}\right)-f\left(\left(r_{i}-\alpha q_{\max }\right)^{+}\right)\right) \\
& -\left(f\left(\left(r_{j}-\alpha q_{\max }\right)^{+}\right)-f\left(\left(r_{j}-\varepsilon-\alpha q_{\max }\right)^{+}\right)\right) \\
\leq & 0
\end{aligned}
$$

by convexity of $f(\cdot)$ since $r_{i}+\varepsilon<r_{j}$. No such improvement exists when $r_{1}=\ldots=r_{\xi}$, in which case we must have $r_{\ell}=H / \xi$ and $\lambda_{\ell}=(\ell-1) H / \xi$ for all $\ell \in\{1, \ldots, \xi\}$ in order to satisfy the condition $\sum_{\ell=1}^{i} r_{\ell}=$ $P\left(\lambda_{i+1}\right)=H \lambda_{i+1}$ for all $i \in\{1, \ldots, \xi\}$. Therefore, the continuous recharging policy $\rho_{P, \xi}^{*}$ is optimal.

\section{Proof of Theorem 2}

For any $\xi$, let $\rho_{P, \xi}^{*}=\left(\left[\lambda_{\ell}^{*}=(\ell-1) H / \xi: \ell=1, \ldots, \xi\right],\left[r_{\ell}^{*}=H / \xi: \ell=1, \ldots, \xi\right]\right)$. By Theorem 1 , this policy is optimal for a given $\xi$. The total cost of the policy $\rho_{P, \xi}^{*}$ is thus

$$
D\left(\rho_{P, \xi}^{*}\right)=\xi s+k\left(H-\xi \alpha q_{\max }\right)^{+} .
$$

We consider the following two cases that describe the possible relations of the recharging amount at each stop, which is the same for all stops, to $\alpha q_{\max }$ :

Case 1: $\alpha q_{\max }<\frac{H}{\xi}$ (i.e., the vehicle always overcharges whenever it stops) and

Case 2: $\alpha q_{\max } \geq \frac{H}{\xi}$ (i.e., the vehicle never overcharges when it stops).

Then for a given case, increasing the value of $\xi$ by one (i.e., adding an extra recharging stop) decreases the amount recharged at each stop to $\frac{H}{\xi+1}$. This results in a transition from Case 1 to Case 2 if $\frac{H}{\xi+1}<\alpha q_{\max } \leq \frac{H}{\xi}$ or no change otherwise. We define the possible transitions as follows:

Transition 1: Case 1 to Case 1,

Transition 2: Case 1 to Case 2, and

Transition 3: Case 2 to Case 2. 
The difference in cost when increasing the number of stops by one is

$$
D\left(\rho_{P, \xi+1}^{*}\right)-D\left(\rho_{P, \xi}^{*}\right)=s- \begin{cases}k \alpha q_{\max }, & \xi \leq \frac{H}{\alpha q_{\max }}-1 \\ k\left(H-\alpha q_{\max }\left\lfloor\frac{H}{\alpha q_{\max }}\right\rfloor\right), & \frac{H}{\alpha q_{\max }}-1<\xi \leq \frac{H}{\alpha q_{\max }} \\ 0, & \xi>\frac{H}{\alpha q_{\max }}\end{cases}
$$

for feasible $\xi$ (i.e., $\xi \geq H / q_{\max }$ ). The subcases (15a), (15b), and (15c) correspond to the cost differences of Transitions 1, 2, and 3, respectively. As $\xi$ increases, note that Transition 1 cannot occur after Transition 2, which in turn cannot occur after Transition 3. We can therefore order the transitions and compare them sequentially. Comparing Transitions 1 and 2 first, we find that

$$
s-k \alpha q_{\max }<s-k\left(H-\alpha q_{\max }\left\lfloor\frac{H}{\alpha q_{\max }}\right\rfloor\right)
$$

since $H-\alpha q_{\max }\left\lfloor H / \alpha q_{\max }\right\rfloor<\alpha q_{\max }$, and comparing Transitions 2 and 3 reveals that

$$
s-k\left(H-\alpha q_{\max }\left\lfloor\frac{H}{\alpha q_{\max }}\right\rfloor\right) \leq s
$$

because $H-\alpha q_{\max }\left\lfloor H / \alpha q_{\max }\right\rfloor \geq 0$. The function $D\left(\rho_{P, \xi}^{*}\right)$ is therefore convex and also piecewise linear with respect to $\xi$ and has up to three segments. We are interested in the value of $\xi$ that minimizes $D\left(\rho_{P, \xi}^{*}\right)$, or the smallest feasible integer value of $\xi$ such that the cost difference is non-negative. There are three different cases to consider.

- If the expression in (15b) is negative, then only the third segment of the function has a non-negative slope. (It is also implied that $\frac{H}{\alpha q_{\max }}$ is non-integer in this case because otherwise we would have $H-\alpha q_{\max }\left\lfloor\frac{H}{\alpha q_{\max }}\right\rfloor=0$ and consequently $s<0$, which is not possible since the stopping time is assumed to be non-negative.) That segment includes values of $\xi$ that are greater than $\frac{H}{\alpha q_{\max }}$, and the smallest integer value of $\xi$ such that $\xi>\frac{H}{\alpha q_{\max }}$, where $\frac{H}{\alpha q_{\max }}$ is non-integer, is $\left\lceil\frac{H}{\alpha q_{\max }}\right\rceil$. This result corresponds to the case $(4 \mathrm{a})$, and because $\left\lceil\frac{H}{\alpha q_{\max }}\right\rceil \geq\left\lceil\frac{H}{q_{\max }}\right\rceil$, where $\left\lceil\frac{H}{q_{\max }}\right\rceil$ is the minimum possible number of stops, it is feasible.

- If the expression in (15a) is negative and the one in $(15 \mathrm{~b})$ is not, then both the second and third segments of the function have non-negative slopes. Thus, the optimal number of stops is included in the second segment (if feasible), which consists of values satisfying $\frac{H}{\alpha q_{\max }}-1<\xi \leq \frac{H}{\alpha q_{\max }}$. The only integer value of $\xi$ that satisfies the inequalities is $\left\lfloor\frac{H}{\alpha q_{\max }}\right\rfloor$, but it cannot be less than the minimum 
possible number of stops, $\left\lceil\frac{H}{q_{\max }}\right\rceil$. This result corresponds to the case (4b).

- If the expression in (15a) is non-negative, then all three segments of the function have non-negative slopes. Therefore, the function is minimized when $\xi$ is minimized, and the smallest feasible integer value of $\xi$ is $\left\lceil\frac{H}{q_{\max }}\right\rceil$. This is also the number of stops made when $\left\lfloor\frac{H}{\alpha q_{\max }}\right\rfloor$ is infeasible for $\xi$ in the previous case, and the result corresponds to the case (4c).

Therefore, the stated expression for $\operatorname{argmin}_{\xi}\left\{D\left(\rho_{P, \xi}^{*}\right)\right\}$ holds.

\section{Proof of Theorem 3}

Suppose for some equidistant recharging policy $\sigma_{P, \xi}=\left(\left[\mu_{1}, \ldots, \mu_{\xi}\right],\left[r_{1}, \ldots, r_{\xi}\right]\right)$ satisfying Property 1 that $r_{i}+h<r_{j}$ for some $i, j \in\{1, \ldots, \xi\}$. Let $\sigma_{P, \xi}^{\prime}=\left(\left[\mu_{1}^{\prime}, \ldots, \mu_{\xi}^{\prime}\right],\left[r_{1}^{\prime}, \ldots, r_{\xi}^{\prime}\right]\right)$ be the policy in which

$$
r_{\ell}^{\prime}= \begin{cases}r_{i}+h, & \ell=i \\ r_{j}-h, & \ell=j \\ r_{\ell}, & \ell \in\{1, \ldots, \xi\} \backslash\{i, j\}\end{cases}
$$

and corresponding $\left[\mu_{1}^{\prime}, \ldots, \mu_{\xi}^{\prime}\right]$ are defined based on Property 1 . Note that this policy is feasible because the total amount recharged along the path is still $n h$ and the vehicle only recharges when its charge level is 0 . Then we have

$$
\begin{aligned}
E\left(\sigma_{P, \xi}^{\prime}\right)-E\left(\sigma_{P, \xi}\right)= & \sum_{\ell=1}^{\xi}\left(c\left(r_{\ell}^{\prime}, 0\right)-c\left(r_{\ell}, 0\right)\right) \\
= & c\left(r_{i}+h, 0\right)-c\left(r_{i}, 0\right)+c\left(r_{j}-h, 0\right)-c\left(r_{j}, 0\right) \\
= & {\left[s+\gamma\left(r_{i}+h\right)+f\left(\left(r_{i}+h-\alpha q_{\max }\right)^{+}\right)\right]-\left[s+\gamma r_{i}+f\left(\left(r_{i}-\alpha q_{\max }\right)^{+}\right)\right] } \\
& +\left[s+\gamma\left(r_{j}-h\right)+f\left(\left(r_{j}-h-\alpha q_{\max }\right)^{+}\right)\right]-\left[s+\gamma r_{j}+f\left(\left(r_{j}-\alpha q_{\max }\right)^{+}\right)\right] \\
= & \left(f\left(\left(r_{i}+h-\alpha q_{\max }\right)^{+}\right)-f\left(\left(r_{i}-\alpha q_{\max }\right)^{+}\right)\right) \\
& -\left(f\left(\left(r_{j}-\alpha q_{\max }\right)^{+}\right)-f\left(\left(r_{j}-h-\alpha q_{\max }\right)^{+}\right)\right) \\
\leq & 0
\end{aligned}
$$

by convexity of $f(\cdot)$ since $r_{i}+h<r_{j}$. No such improvement exists when $\max _{i, j \in\{1, \ldots, \xi\}}\left\{r_{i}-r_{j}\right\} \leq h$, and the total cost cannot be further reduced by violating Property 1 . Therefore, the equidistant recharging policy $\sigma_{P, \xi}^{*}$ is optimal. 


\section{Proof of Theorem 4}

Let $\sigma_{P, \xi}^{*}=\left(\left[\mu_{1}^{*}, \ldots, \mu_{\xi}^{*}\right],\left[r_{1}^{*}, \ldots, r_{\xi}^{*}\right]\right)$ with $r_{1}^{*}=\ldots=r_{n-\xi\left\lfloor\frac{n}{\xi}\right\rfloor}^{*}=\left\lceil\frac{n}{\xi}\right\rceil h$ and $r_{n-\xi\left\lfloor\frac{n}{\xi}\right\rfloor+1}^{*}=\ldots=r_{\xi}^{*}=\left\lfloor\frac{n}{\xi}\right\rfloor h$. By Theorem 3, this policy is optimal for a given $\xi$. The total cost of policy $\sigma_{P, \xi}^{*}$ is thus

$$
E\left(\sigma_{P, \xi}^{*}\right)=\xi s+k \sum_{i=1}^{\xi}\left(r_{i}^{*}-\alpha q_{\max }\right)^{+}
$$

If we relate the two possible values for the amount to recharge at each stop to $\alpha q_{\max }$, we see that there are three possible cases to consider:

Case 1: $\alpha q_{\max }<\left\lfloor\frac{n}{\xi}\right\rfloor h \leq\left\lceil\frac{n}{\xi}\right\rceil h$ (i.e., the vehicle always overcharges whenever it stops),

Case 2: $\left\lfloor\frac{n}{\xi}\right\rfloor h \leq \alpha q_{\max }<\left\lceil\frac{n}{\xi}\right\rceil h$ (i.e., the vehicle overcharges at the first $n-\xi\left\lfloor\frac{n}{\xi}\right\rfloor$ stops but not at the remaining stops), and

Case 3: $\left\lfloor\frac{n}{\xi}\right\rfloor h \leq\left\lceil\frac{n}{\xi}\right\rceil h \leq \alpha q_{\max }$ (i.e., the vehicle never overcharges when it stops).

Then for a given case, increasing the value of $\xi$ by one (i.e., adding an extra recharging stop) decreases the amounts recharged to $\left\lfloor\frac{n}{\xi+1}\right\rfloor h$ and $\left\lceil\frac{n}{\xi+1}\right\rceil h$ for the corresponding stops. This could result in a new relationship of the recharging amounts to $\alpha q_{\max }$ and transition us to a different case. For example, we transition from Case 1 to Case 2 when $\alpha q_{\max }<\left\lfloor\frac{n}{\xi}\right\rfloor h \leq\left\lceil\frac{n}{\xi}\right\rceil h$ and $\left\lfloor\frac{n}{\xi+1}\right\rfloor h \leq \alpha q_{\max }<\left\lceil\frac{n}{\xi+1}\right\rceil h$. The possible transitions are the following:

Transition 1: Case 1 to Case 1,

Transition 2: Case 1 to Case 2,

Transition 3: Case 2 to Case 2,

Transition 4: Case 1 to Case 3,

Transition 5: Case 2 to Case 3, and

Transition 6: Case 3 to Case 3.

Note that $\frac{n}{y+1}$ is the maximum (possibly fractional) number of stops such that the amount recharged at each stop is greater than $\alpha q_{\max }$, and $\left\lfloor\frac{n}{y}\right\rfloor$ is the maximum feasible number of stops such that not all of the amounts recharged at each stop are less than $\alpha q_{\max }$. If $\frac{n}{y+1}>\left\lfloor\frac{n}{y}\right\rfloor$, then there is no feasible value for $\xi$ such that $\left\lfloor\frac{n}{\xi}\right\rfloor h \leq \alpha q_{\max }<\left\lceil\frac{n}{\xi}\right\rceil h$. Thus, we only need to consider Transitions 1, 4, and 6. Alternatively, if 
$\frac{n}{y+1} \leq\left\lfloor\frac{n}{y}\right\rfloor$, then all transitions except Transition 4 are possible. We now compute the costs for each of the six possible transitions.

The difference in cost when increasing the number of stops by one is

$$
\begin{aligned}
& E\left(\sigma_{P, \xi+1}^{*}\right)-E\left(\sigma_{P, \xi}^{*}\right)= \\
& s- \begin{cases}k \alpha q_{\max }, & \xi<\left\lfloor\frac{n}{y+1}\right\rfloor \\
k\left(\alpha q_{\max }-\left((y+1)\left\lceil\frac{n}{y+1}\right]-n\right)\left(\alpha q_{\max }-y h\right)\right), & \xi=\left\lfloor\frac{n}{y+1}\right\rfloor<\frac{n}{y+1} \text { and } \frac{n}{y+1} \leq\left\lfloor\frac{n}{y}\right\rfloor \\
k y\left((y+1) h-\alpha q_{\max }\right), & \left\lceil\frac{n}{y+1}\right\rfloor \leq \xi<\left\lfloor\frac{n}{y}\right\rfloor \text { and } \frac{n}{y+1} \leq\left\lfloor\frac{n}{y}\right\rfloor \\
k\left(n h-\left\lfloor\frac{n}{y}\right\rfloor \alpha q_{\max }\right), & \xi=\left\lfloor\frac{n}{y}\right\rfloor<\frac{n}{y} \text { and } \frac{n}{y+1}>\left\lfloor\frac{n}{y}\right\rfloor \\
k\left(n-y\left\lfloor\frac{n}{y}\right\rfloor\right)\left((y+1) h-\alpha q_{\max }\right), & \xi=\left\lfloor\frac{n}{y}\right\rfloor<\frac{n}{y} \text { and } \frac{n}{y+1} \leq\left\lfloor\frac{n}{y}\right\rfloor \\
0, & \xi \geq\left\lceil\frac{n}{y}\right\rfloor\end{cases}
\end{aligned}
$$

for feasible $\xi$ (i.e., $\xi \geq H / q_{\max }$ ). The six subcases in (17) correspond to the cost differences of Transitions 1 through 6 , in order.

We first consider the case where $\frac{n}{y+1}>\left\lfloor\frac{n}{y}\right\rfloor$. As $\xi$ increases, note that Transition 1 cannot occur after Transition 4, which in turn cannot occur after Transition 6 . We can therefore order the transitions and compare them sequentially. Beginning with Transitions 1 and 4, we compare (17a) and (17d) and find that

$$
\begin{aligned}
s-k \alpha q_{\max } & \leq s-k\left(\left(n-y\left\lfloor\frac{n}{y}\right\rfloor\right) h-\left\lfloor\frac{n}{y}\right\rfloor\left(\alpha q_{\max }-y h\right)\right) \\
& =s-k\left(n h-\left\lfloor\frac{n}{y}\right\rfloor \alpha q_{\max }\right)
\end{aligned}
$$

since $\left(n-y\left\lfloor\frac{n}{y}\right\rfloor\right) h<y h \leq \alpha q_{\max }$ and $\left\lfloor\frac{n}{y}\right\rfloor\left(\alpha q_{\max }-y h\right) \geq 0$. Comparing Transitions 4 and 6 , we note that

$$
s-k\left(n h-\left\lfloor\frac{n}{y}\right\rfloor \alpha q_{\max }\right)<s-k\left(n h-\frac{n}{y+1}(y+1) h\right)=s
$$

because $\frac{n}{y+1}>\left\lfloor\frac{n}{y}\right\rfloor$ and $(y+1) h>\alpha q_{\max }$. Thus, $E(\cdot)$ is convex and piecewise linear with respect to $\xi$ in this case.

We next consider the case where $\frac{n}{y+1} \leq\left\lfloor\frac{n}{y}\right\rfloor$. As in the previous case, the transitions can be ordered and compared sequentially. We first compare (17a) and (17b) (Transitions 1 and 2) and see that

$$
s-k \alpha q_{\max } \leq k\left(\alpha q_{\max }-\left((y+1)\left\lceil\frac{n}{y+1}\right\rceil-n\right)\left(\alpha q_{\max }-y h\right)\right)
$$


because $(y+1)\left\lceil\frac{n}{y+1}\right\rceil-n \geq 0$ and $\alpha q_{\max }-y h \geq 0$. We next compare Transitions 2 and 3 and find that

$$
\begin{aligned}
s-k\left(\alpha q_{\max }-\left((y+1)\left\lceil\frac{n}{y+1}\right\rceil-n\right)\left(\alpha q_{\max }-y h\right)\right) & \leq s-k\left(\alpha q_{\max }-(y+1)\left(\alpha q_{\max }-y h\right)\right) \\
& =s-k y\left((y+1) h-\alpha q_{\max }\right) .
\end{aligned}
$$

Comparing (17c) and (17e), or Transitions 3 and 5, gives

$$
s-k y\left((y+1) h-\alpha q_{\max }\right)<s-k\left(n-y\left\lfloor\frac{n}{y}\right\rfloor\right)\left((y+1) h-\alpha q_{\max }\right)
$$

and the expression in (17e) is no greater than $s$, the cost of Transition 6 . Therefore, in each of the cases $\frac{n}{y+1}>\left\lfloor\frac{n}{y}\right\rfloor$ and $\frac{n}{y+1} \leq\left\lfloor\frac{n}{y}\right\rfloor$, the function $E(\cdot)$ is convex and also piecewise linear with respect to $\xi$. We are interested in the value of $\xi$ that minimizes $E(\cdot)$, or the smallest feasible integer of $\xi$ such that the cost difference is non-negative. There are several cases to consider.

- If the expression in (17d) is negative when $\frac{n}{y+1}>\left\lfloor\frac{n}{y}\right\rfloor$ or the expression in (17e) is negative when $\frac{n}{y+1} \leq\left\lfloor\frac{n}{y}\right\rfloor$, then only the last segment of the function has a non-negative slope. That segment includes values of $\xi$ that are at least $\left\lceil\frac{n}{y}\right\rceil$, and the smallest integer value of $\xi$ such that $\xi \geq\left\lceil\frac{n}{y}\right\rceil$ is $\left\lceil\frac{n}{y}\right\rceil$. This result corresponds to cases (5a) and (6a), and because $\left\lceil\frac{n}{y}\right\rceil \geq\left\lceil\frac{n}{\left\lfloor q_{\max } / h\right\rfloor}\right\rceil$, where $\left\lceil\frac{n}{\left\lfloor q_{\max } / h\right\rfloor}\right\rceil$ is the minimum possible number of stops, it is feasible.

- If the expression in (17a) is negative and the one in (17d) is not when $\frac{n}{y+1}>\left\lfloor\frac{n}{y}\right\rfloor$, or if the expression in (17c) is negative and the one in (17e) is not when $\frac{n}{y+1} \leq\left\lfloor\frac{n}{y}\right\rfloor$, then if $\left\lfloor\frac{n}{y}\right\rfloor$ is a feasible number of stops, the last two segments of the function have non-negative slopes. Thus, the optimal number of stops is included in the second-to-last segment, which consists of values satisfying $\xi=\left\lfloor\frac{n}{y}\right\rfloor<\frac{n}{y}$. The only integer value of $\xi$ that satisfies the expression is $\left\lfloor\frac{n}{y}\right\rfloor$, where $\frac{n}{y}$ must be non-integer. This result corresponds to cases $(5 \mathrm{~b})$ and $(6 \mathrm{~b})$.

- If the expression in (17b) is negative and the one in (17c) is not when $\frac{n}{y+1} \leq\left\lfloor\frac{n}{y}\right\rfloor$, then the third, fourth, and fifth segments of the function have non-negative slopes. The optimal number of stops is therefore included in the third segment (if feasible) when $\frac{n}{y+1} \leq\left\lfloor\frac{n}{y}\right\rfloor$, which consists of values satisfying $\left\lceil\frac{n}{y+1}\right\rceil \leq \xi<\left\lfloor\frac{n}{y}\right\rfloor$. The smallest integer value of $\xi$ satisfying the expression is $\left\lceil\frac{n}{y+1}\right\rceil$, but it must be feasible and cannot be less than $\left\lceil\frac{n}{\left[q_{\max } / h\right]}\right\rceil$. This result corresponds to case (6c).

- If the expression in (17a) is negative and the one in (17b) is not when $\frac{n}{y+1} \leq\left\lfloor\frac{n}{y}\right\rfloor$, and if $\left\lfloor\frac{n}{y+1}\right\rfloor$ is 
a feasible number of stops, then the second through fifth segments of the function have non-negative slopes. Thus, the optimal number of stops is included in the second segment when $\frac{n}{y+1} \leq\left\lfloor\frac{n}{y}\right\rfloor$, which consists of values satisfying $\xi=\left\lfloor\frac{n}{y+1}\right\rfloor<\frac{n}{y+1}$. The only integer value of $\xi$ that satisfies the expression is $\left\lfloor\frac{n}{y+1}\right\rfloor$, where $\frac{n}{y+1}$ must be non-integer. This result corresponds to case $(6 \mathrm{~d})$.

- If the expression in (17a) is non-negative, then all segments of the function have non-negative slopes. Therefore, the function is minimized when $\xi$ is minimized, and the smallest feasible integer value of $\xi$ is $\left\lceil\frac{n}{\left[q_{\max } / h\right]}\right]$. This is also the number of stops that is made if the value in any of the previous cases is infeasible, and the result corresponds to cases (5c) and (6e).

Therefore, the stated expressions for $\operatorname{argmin}_{\xi}\left\{E\left(\sigma_{P, \xi}^{*}\right)\right\}$ hold.

\section{Proof of Theorem 5}

Let $K$ denote the overcharging cost incurred by the policy $\hat{\pi}_{P}$, where

$$
K=\sum_{i=1}^{n} f\left(\left(h_{i}-\alpha q_{\max }\right)^{+}\right)
$$

since the policy only overcharges whenever $h_{i}>\alpha q_{\max }$. Then the total cost of the policy $\hat{\pi}_{P}$ satisfies

$$
C\left(\hat{\pi}_{P}\right)=\hat{\xi} s+K \leq\left\lceil\frac{2 H}{\alpha q_{\max }}\right\rceil s+K \quad(\text { by Lemma } 4)
$$

It follows that the ratio of the cost of the heuristic policy to the cost of an optimal policy must satisfy

$$
\frac{C\left(\hat{\pi}_{P}\right)}{C\left(\pi_{P}^{*}\right)} \leq \frac{\left\lceil\frac{2 H}{\alpha q_{\max }}\right\rceil s+K}{\left\lceil\frac{H}{q_{\max }}\right\rceil s+\sum_{i=1}^{n} f\left(\left(r_{i}^{*}-\alpha q_{\max }\right)^{+}\right)} \leq \frac{\left\lceil\frac{2 H}{\alpha q_{\max }}\right\rceil s+K}{\frac{H}{q_{\max }} s+K}
$$

because any feasible recharging policy must incur overcharging costs of at least $K$. Furthermore, since $\left\lceil\frac{2 H}{\alpha q_{\max }}\right\rceil s>\frac{H}{q_{\max }} s$, it follows that

$$
\frac{C\left(\hat{\pi}_{P}\right)}{C\left(\pi_{P}^{*}\right)} \leq \frac{\left\lceil\frac{2 H}{\alpha q_{\max }}\right\rceil}{\frac{H}{q_{\max }}}<\frac{\frac{2 H}{\alpha q_{\max }}+1}{\frac{H}{q_{\max }}}=\frac{2}{\alpha}+\frac{q_{\max }}{H}
$$

as desired. 


\section{Proof of Theorem 6}

By extension of Lemma 3, it can be seen that the policy $\tilde{\pi}_{P}$ minimizes the number of recharging stops along $P$. For $\alpha=1$, the lemma states that the policy $\tilde{\pi}_{P}$ is optimal, and its corresponding cost would be $\tilde{\xi} s$, where $\tilde{\xi}$ is the number of stops in the policy. Therefore, $\tilde{\xi}$ must be the minimum feasible number of stops. For the case where $\alpha<1$, however, the policy may not be optimal due to the possibility of overcharging costs. At most, the amount overcharged along the entire path is $(1-\alpha) H$. If we let $\xi^{*}$ denote the number of stops in the optimal policy $\pi_{P}^{*}$, then we have

$$
\frac{C\left(\tilde{\pi}_{P}\right)}{C\left(\pi_{P}^{*}\right)} \leq \frac{\tilde{\xi} s+k(1-\alpha) H}{\xi^{*} s+k\left(H-\xi^{*} \alpha q_{\max }\right)^{+}}
$$

For $s / k<\alpha q_{\max }$, the cost of an optimal policy is bounded below by $\left\lfloor\frac{H}{\alpha q_{\max }}\right\rfloor s$, which comes from the continuous result given in Theorem 2. It follows that

$$
\begin{aligned}
\frac{C\left(\tilde{\pi}_{P}\right)}{C\left(\pi_{P}^{*}\right)} & \leq 1+\frac{k(1-\alpha) H}{\xi^{*} s+k\left(H-\xi^{*} \alpha q_{\max }\right)^{+}} \quad\left(\text { since } \tilde{\xi} \leq \xi^{*}\right) \\
& \leq 1+\frac{k(1-\alpha) H}{\left\lfloor\frac{H}{\alpha q_{\max }}\right\rfloor s} \\
& =1+(1-\alpha)\left(\frac{H / \alpha q_{\max }}{\left\lfloor H / \alpha q_{\max }\right\rfloor}\right)\left(\frac{\alpha q_{\max }}{s / k}\right) .
\end{aligned}
$$

When $s / k \geq \alpha q_{\max }$, the cost of an optimal policy is bounded below by $\left\lceil\frac{H}{q_{\max }}\right\rceil s+k\left(H-\left\lceil\frac{H}{q_{\max }}\right\rceil \alpha q_{\max }\right)^{+}$ (from Theorem 2), and therefore

$$
\begin{aligned}
& \frac{C\left(\tilde{\pi}_{P}\right)}{C\left(\pi_{P}^{*}\right)} \leq 1+\frac{k(1-\alpha) H-k\left(H-\xi^{*} \alpha q_{\max }\right)^{+}}{\xi^{*} s+k\left(H-\xi^{*} \alpha q_{\max }\right)^{+}} \quad\left(\text { since } \tilde{\xi} \leq \xi^{*}\right) \\
& \leq 1+\frac{k(1-\alpha) H-k\left(H-\left\lceil\frac{H}{q_{\max }}\right\rceil \alpha q_{\max }\right)^{+}}{\left\lceil\frac{H}{q_{\max }}\right\rceil s+k\left(H-\left\lceil\frac{H}{q_{\max }}\right\rceil \alpha q_{\max }\right)^{+}} \\
& \leq 1+\frac{(1-\alpha) H-\left(H-\left\lceil\frac{H}{q_{\max }}\right\rceil \alpha q_{\max }\right)^{+}}{\left\lceil\frac{H}{q_{\max }}\right\rceil \alpha q_{\max }+\left(H-\left\lceil\frac{H}{q_{\max }}\right\rceil \alpha q_{\max }\right)^{+}} \quad\left(\text { since } s / k \geq \alpha q_{\max }\right) \\
& =1+ \begin{cases}\frac{(1-\alpha) H}{\left\lceil\frac{H}{q_{\max }}\right\rceil \alpha q_{\max }}, & H-\left\lceil\frac{H}{q_{\max }}\right\rceil \alpha q_{\max } \leq 0 \\
\alpha\left(\left\lceil\frac{H}{q_{\max }}\right\rceil\left(\frac{q_{\max }}{H}\right)-1\right), & H-\left\lceil\frac{H}{q_{\max }}\right\rceil \alpha q_{\max }>0\end{cases}
\end{aligned}
$$




$$
\leq \begin{cases}1 / \alpha, & H-\left\lceil\frac{H}{q_{\max }}\right\rceil \alpha q_{\max } \leq 0 \\ 1+\frac{\alpha q_{\max }}{H}, & H-\left\lceil\frac{H}{q_{\max }}\right\rceil \alpha q_{\max }>0\end{cases}
$$

Thus, the stated bound for $C\left(\tilde{\pi}_{P}\right) / C\left(\pi_{P}^{*}\right)$ is valid. 


\section{Appendix A2: Supplemental figures}

\section{Heuristic performance for trips longer than 300 miles}
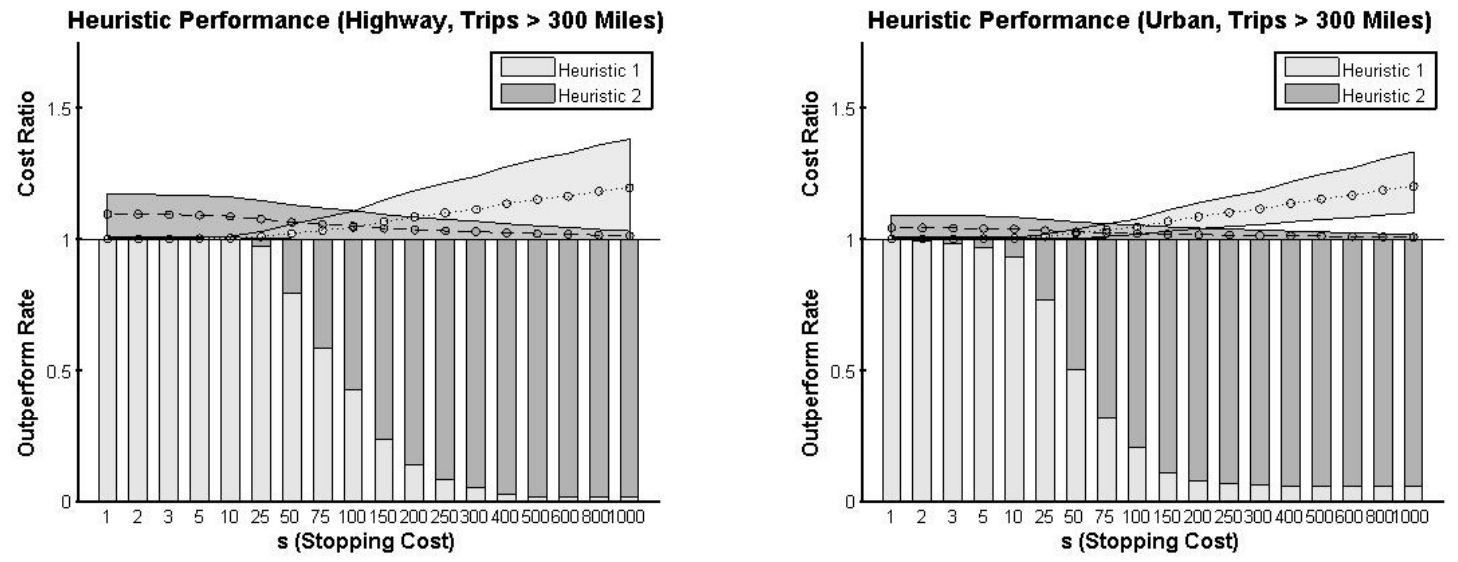

Figure 17: Heuristic recharging policy performance for trips longer than 300 miles; mean Cost Ratios with 10th-90th interpercentile ranges are shown 